Please do not remove this page

RMIT

UNIVERSITY

\title{
LIDAR obstacle warning and avoidance system for unmanned aerial vehicle sense-and-avoid
}

Ramasamy, Subramanian; Sabatini, Roberto; Gardi, Alex; Liu, Jing

https://researchrepository.rmit.edu.au/esploro/outputs/9921860499701341/filesAndLinks?institution=61RMIT_INST\&index=null

Ramasamy, S., Sabatini, R., Gardi, A., \& Liu, J. (2016). LIDAR obstacle warning and avoidance system for unmanned aerial vehicle sense-and-avoid. Aerospace Science and Technology, 55, 344-358.

https://doi.org/10.1016/j.ast.2016.05.020

Document Version: Accepted Manuscript

Published Version: https://doi.org/10.1016/j.ast.2016.05.020

Repository homepage: https://researchrepository.rmit.edu.au

(C) 2016 Elsevier Masson SAS. All rights reserved

Downloaded On 2023/04/26 23:30:30 +1000

Please do not remove this page 
Thank you for downloading this document from the RMIT Research Repository.

The RMIT Research Repository is an open access database showcasing the research outputs of RMIT University researchers.

RMIT Research Repository: http://researchbank.rmit.edu.au/

\section{Citation:}

Ramasamy, S, Sabatini, R, Gardi, A and Liu, J 2016, 'LIDAR obstacle warning and avoidance system for unmanned aerial vehicle sense-and-avoid', Aerospace Science and Technology, vol. 55, pp. 344-358.

See this record in the RMIT Research Repository at:

https://researchbank.rmit.edu.au/view/rmit:37299

Version: Accepted Manuscript

Copyright Statement: (C)2016 Elsevier Masson SAS. All rights reserved Creative Commons Attribution-NonCommercial-NoDerivatives 4.0 International License.

Link to Published Version:

http://dx.doi.org/10.1016/j.ast.2016.05.020 


\title{
LIDAR Obstacle Warning and Avoidance System for Unmanned Aerial Vehicle Sense-and-Avoid
}

\author{
Subramanian Ramasamy, Roberto Sabatini ${ }^{*}$ *, Alessandro Gardi, Jing Liu \\ School of Engineering,-Aerospace and Aviation Discipline, RMIT University, Melbourne, VIC 3000, Australia
}

\begin{abstract}
The demand for reliable obstacle warning and avoidance capabilities to ensure safe low-level flight operations has led to the development of various practical systems suitable for fixed and rotary wing aircraft. State-of-the-art Light Detection and Ranging (LIDAR) technology employing eye-safe laser sources, advanced electro-optics and mechanical beam-steering components delivers the highest angular resolution and accuracy performances in a wide range of operational conditions. LIDAR Obstacle Warning and Avoidance System (LOWAS) is thus becoming a mature technology with several potential applications to manned and unmanned aircraft. This paper addresses specifically its employment in Unmanned Aircraft Systems (UAS) Sense-and-Avoid (SAA). Small-to-medium size Unmanned Aerial Vehicles (UAVs) are particularly targeted since they are very frequently operated in proximity of the ground and the possibility of a collision is further aggravated by the very limited see-and-avoid capabilities of the remote pilot. After a brief description of the system architecture, mathematical models and algorithms for avoidance trajectory generation are provided. Key aspects of the Human Machine Interface and Interaction $\left(\mathrm{HMI}^{2}\right)$ design for the UAS obstacle avoidance system are also addressed. Additionally, a comprehensive simulation case study of the avoidance trajectory generation algorithms is presented. It is concluded that LOWAS obstacle detection and trajectory optimisation algorithms can ensure a safe avoidance of all classes of obstacles (i.e., wire, extended and point objects) in a wide range of weather and geometric conditions, providing a pathway for possible integration of this technology into future UAS SAA architectures.
\end{abstract}

Keywords:

Airborne Lasers; Laser Sensors; Integrated Avionics Systems; Obstacle Warning and Avoidance; Sense-and-Avoid; Unmanned Aerial Vehicle.

\section{Introduction}

A number of Unmanned Aerial Vehicle (UAV) mission-andsafety critical tasks involve low-level flight activities beyond the relatively safe aerodrome perimeter. Low level and terrainfollowing operations are often challenged by a variety of natural and man-made obstacles. The significant number of obstacle strike accidents recorded is a major concern both for aircraft operators and for people on the ground [1, 2]. Reduced atmospheric visibility due to adverse weather conditions is frequently a contributing factor in such accidents, but the difficult identification of small-size obstacles such as wires has led to accidents and incidents even in clear sky conditions. Significant development activities are specifically addressing the integration of obstacle detection, warning and avoidance systems for granting separation maintenance and collision avoidance capabilities [3-9]. Table 1 compares a number of sensor technologies for Obstacle Warning System (OWS) applications in small-to-medium size UAVs. Unfortunately, state-of-the-art radar is not capable of detecting small natural and man-made obstacles such as trees, power line cables and poles. The outstanding angular resolution and accuracy characteristics of Light Detection and Ranging (LIDAR), as well as its good detection performance in a wide range of incidence angles and weather conditions provide an ideal solution for obstacle detection and avoidance [2].

One of the key challenges encountered by the aviation community for integration of Unmanned Aircraft Systems (UAS) into nonsegregated airspace is the provision of a certifiable Sense-andAvoid (SAA) capability. SAA can be defined as the automatic detection of possible conflicts by the UAS and the resolution of any existing collision threats by accomplishing safe avoidance manoeuvres. The maturity of SAA techniques and enabling technologies is considered low when viewed in the perspective of civil airworthiness regulations for manned aircraft, raising concerns to certification authorities and airspace users [8]. With the growing adoption of UAS for a number of civil, commercial and scientific applications, there is a need to certify UAS according to established national and international standards [9]. Such SAA systems will provide UAS the capability to consistently and reliably perform equally or even to exceed the see-and-avoid performance of a human pilot in manned aircraft while allowing a seamless integration of unmanned aircraft in the Air Traffic Management (ATM) network. Research efforts are primarily concentrated on adopting LIDAR sensors for small-tomedium size UAV platforms as one of the most accurate noncooperative SAA sensors. The LIDAR Obstacle Warning and Avoidance System (LOWAS) for UAS is a low-weight/volume navigation aid system specifically designed to detect potentially dangerous ground and aerial obstacles placed in or nearby the planned flight trajectory and to provide timely warnings to the crew in order to implement effective avoidance manoeuvres.

This paper presents the LOWAS hardware and software architecture for UAV applications, including algorithms for automated obstacle avoidance and Human Machine Interface and Interaction $\left(\mathrm{HMI}^{2}\right)$ including synthetic display formats for the UAS Ground Control Station (GCS). A key novelty aspect of the LOWAS development is in the analytical models implemented for real-time processing of navigation and tracking errors affecting the state measurements allowing a direct translation into unified range and bearing uncertainty descriptors. Since these errors may be statistically independent (e.g., non-cooperative SAA) or dependent (e.g., cooperative SAA), the uncertainty volume is calculated in real-time for each obstacle encounter. Based on this uncertainty volume, appropriate geo-fences are dynamically generated (whose characteristics are dictated by the obstacle classification), to allow computation of the optimal avoidance flight trajectories. 
This is the author pre-publication version. This paper does not include the changes arising from the revision, formatting and publishing process. The final paper that should be used for referencing is:

S. Ramasamy, R. Sabatini, A. Gardi and J. Liu, "LIDAR Obstacle Warning and Avoidance System for Unmanned Aerial Vehicle Sense-andAvoid.” Aerospace Science and Technology (Elsevier), vol. 55, pages 344-358, 2016. DOI: 10.1016/j.ast.2016.05.020

Table 1. Obstacle detection technologies.

\begin{tabular}{|c|c|c|c|c|}
\hline REQUIREMENT & MAGNETIC & THERMAL & $\begin{array}{c}\text { MILLIMETRIC } \\
\text { WAVE RADAR }\end{array}$ & LIDAR \\
\hline Wire detection & $\begin{array}{c}\text { Only energized } \\
\text { wires }\end{array}$ & Only energized wires & $\begin{array}{c}\text { All wires preferably } \\
\text { perpendicular to flight } \\
\text { trajectory }\end{array}$ & As required \\
\hline Detection range & Short & Short & As required & As required \\
\hline $\begin{array}{c}\text { Coverage Area } \\
\text { Resolution and accuracy } \\
\text { distance) }\end{array}$ & Small & As required & Very high \\
\hline $\begin{array}{c}\text { All-weather performance in } \\
\text { low-level flight }\end{array}$ & Good & $\begin{array}{c}\text { Good for position and } \\
\text { type, no ranging } \\
\text { capabilities }\end{array}$ & Very good & Good* \\
\hline False alarm rate & High & Mature & Moor & Very low \\
\hline Base technology status & State-of-the-art & State-of-the-art \\
\hline
\end{tabular}

* Laser energy is significantly attenuated by rain and blocked by clouds and fog.

\section{Operational requirements}

The OWS operational requirements include:

- Capability to detect all types of hazardous obstacles, including topographic features, vegetation, buildings, poles/masts, towers, cables and transmission lines;

- Operability in all-time and all-weather conditions, including low-light and darkness;

- High minimum detection range, adequate for the platform velocity and dynamic performances;

- Wide Field of View (FOV), adequate for the manoeuvring envelope limits of the platform;

- High range and bearing resolution;

- Accurate and good probability of detection, since no real obstacle threat shall remain undetected;

- Very low false alarm rate, to prevent spurious warnings that would increase the remote pilot's workload and prompt unnecessary avoidance manoeuvres, potentially disruptive to both safety and effectiveness of the mission;

- Satisfactory technological readiness levels.

\section{System description}

LOWAS is designed to detect obstacles placed in or nearby the aircraft trajectory, classify/prioritise the detected obstacles and provide visual and aural warnings and information to the crew. The key components of LOWAS are the Sensor Head Unit (SHU), the Processing Unit (PU), the Control Panel (CP) and the Display Unit (DU). The LIDAR beam scans periodically the area around the host platform's longitudinal axis within a FOV of $40^{\circ}$ in azimuth and $30^{\circ}$ in elevation (Fig. 1).

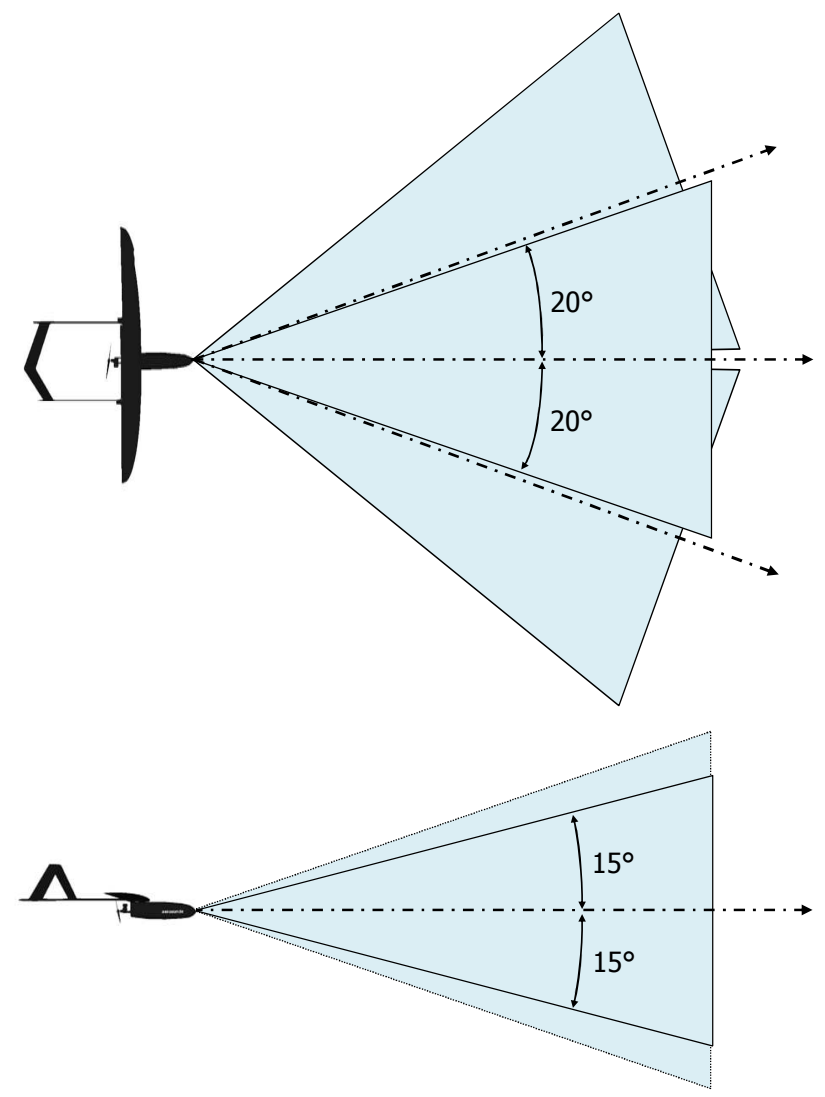

Fig. 1. LOWAS FOV

In order to enhance coverage during turning manoeuvres at high yawing rates, the remote pilot may vary the azimuth orientation of the LOWAS FOV by $20^{\circ}$ left/right with respect to the vertical axis. As conceptually depicted in Fig. 2, during every complete FOV scan (4 Hz refresh frequency), the LIDAR beam generates a number of elliptical scan patterns across the FOV. 
This is the author pre-publication version. This paper does not include the changes arising from the revision, formatting and publishing process. The final paper that should be used for referencing is:

S. Ramasamy, R. Sabatini, A. Gardi and J. Liu, "LIDAR Obstacle Warning and Avoidance System for Unmanned Aerial Vehicle Sense-andAvoid.” Aerospace Science and Technology (Elsevier), vol. 55, pages 344-358, 2016. DOI: 10.1016/j.ast.2016.05.020

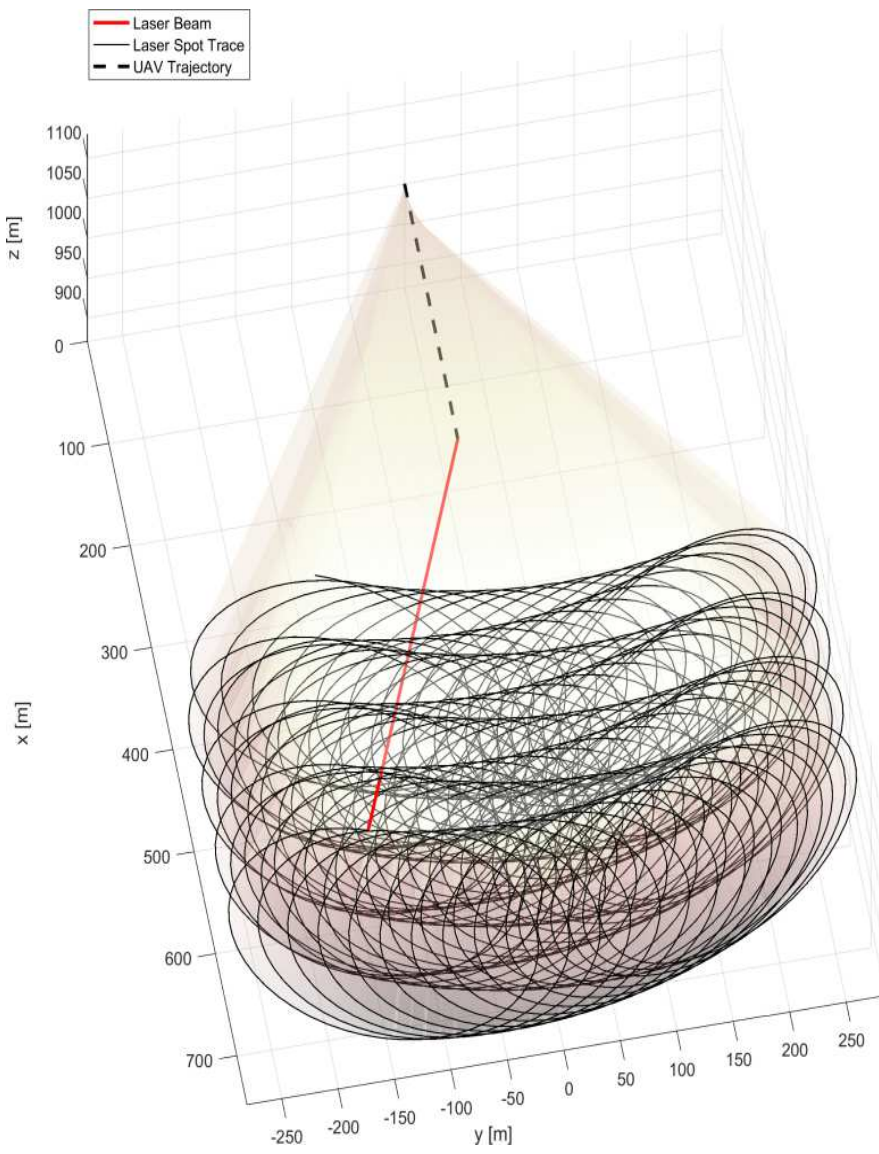

This scanning pattern is well suited to detect the most dangerous obstacles like wires as it produces several and regularly spaced vertical lines. The electro-mechanical device that is used to produce the described scanning pattern is a swashing mirror. The LOWAS laser is the IRE POLUS model ELPM-20K, whose main characteristics are listed in Table 2 [2]. The wavelength, power and pulse duration were chosen to meet eye-safety requirements $[10,11]$. The LOWAS architecture for UAV integration is shown in Fig. 3. In manned aircraft, LOWAS display unit and warning generator are located in the cockpit, while in the case of an UAV, the interactions with remote pilot involves Line-of-Sight (LOS) and Beyond LOS (BLOS) communication links. Both LOS and BLOS data links are necessary for LOWAS communication with the GCS and with the Air Traffic Management (ATM) system. Telemetry data need to be exchanged between the UAS and the GCS for aircraft control and downlinking of both flight parameters and obstacle information (enabling vehicle tracking, mission control and mission profile updates).

Table 2. ELPM-20K laser parameters [2].

\begin{tabular}{|l|c|}
\hline Parameter & Value \\
\hline Emission wavelength & $1.55 \mu \mathrm{m}$ \\
\hline Pulse power at the assembly output & $10 \mathrm{~kW}$ \\
\hline Pulse Duration & $2.8 \mathrm{~ns}$ \\
\hline Pulse Repetition Frequency & $40 \mathrm{kHz}$ \\
\hline
\end{tabular}

Fig. 2. LOWAS scan pattern for an advancing UAV platform.

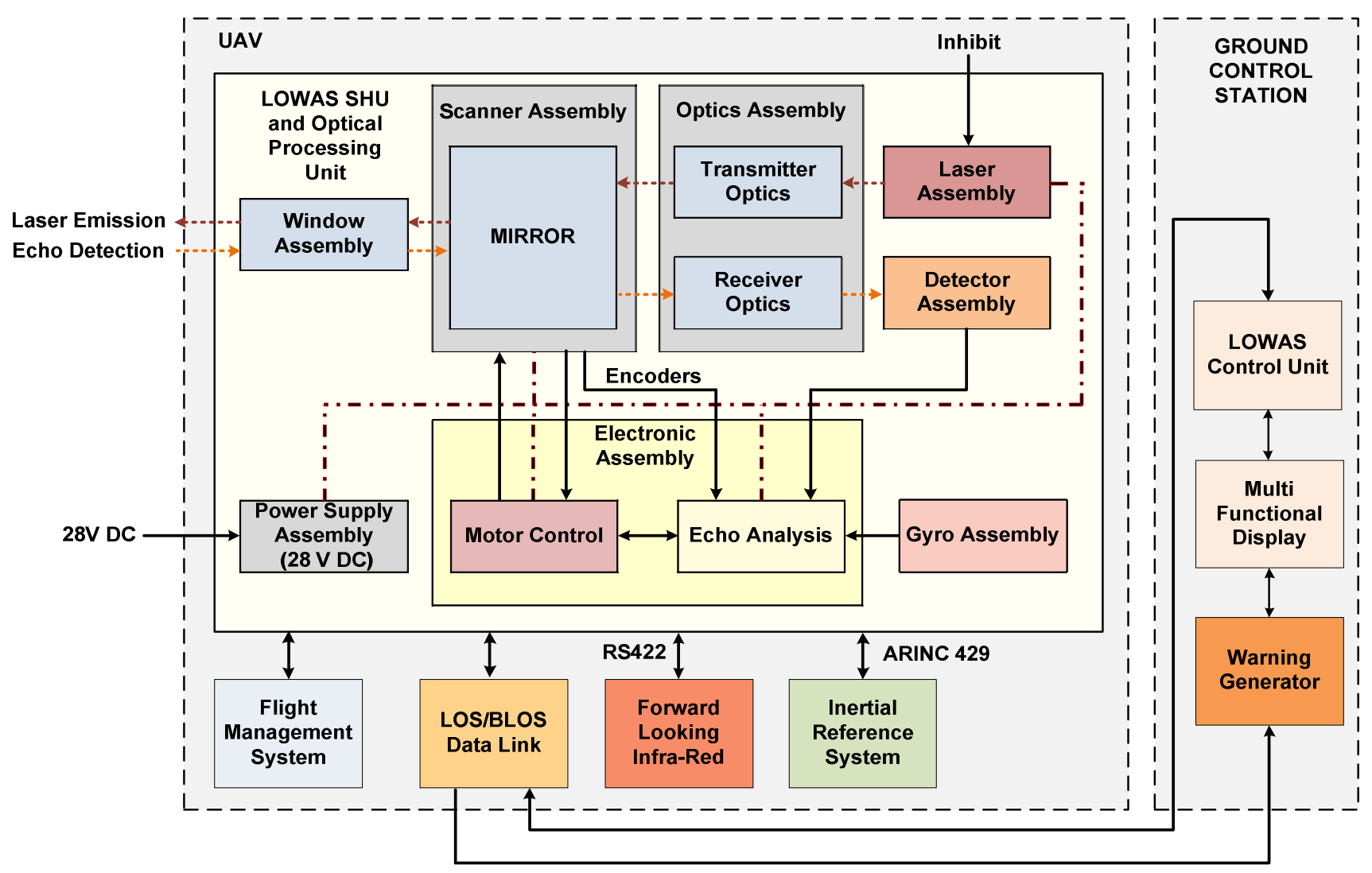

Fig. 3. LOWAS avionics integration architecture for unmanned aircraft. 
This is the author pre-publication version. This paper does not include the changes arising from the revision, formatting and publishing process. The final paper that should be used for referencing is:

S. Ramasamy, R. Sabatini, A. Gardi and J. Liu, "LIDAR Obstacle Warning and Avoidance System for Unmanned Aerial Vehicle Sense-andAvoid.” Aerospace Science and Technology (Elsevier), vol. 55, pages 344-358, 2016. DOI: 10.1016/j.ast.2016.05.020

LOWAS employs three key algorithms namely: prediction of the future platform trajectory; calculation of the potential collisions with the detected obstacles; and generation of a set of optimal avoidance trajectories (in case a risk of collision is determined).

\section{Obstacle detection and classification software architecture}

The signal pre-processing steps involve an analogue opticalelectrical conversion of the echo signal by an Avalanche Photodiode (APD), a signal pre-amplification by an Automatic Gain Control (AGC) and a comparison with adjustable threshold in order to fine-tune sensitivity on the basis of the expected return signal power in relation with the time elapsed from the LIDAR pulse emission. The threshold level may also be tuned to take into account the background conditions. These features reduce the probability of false echo detection due to atmospheric backscattering near the laser beam output and optimise the system sensitivity in all weather conditions. Subsequently, digital signal processing is performed in order to validate positive echo detections, to determine the position of the detected obstacles and to extract their geometrical characteristics. For this purpose, the LOWAS software architecture is organised in two sequential stages: Low Level Processing (LLP) and High Level Processing (HLP). Fig. 4 represents the signal processing software architecture.

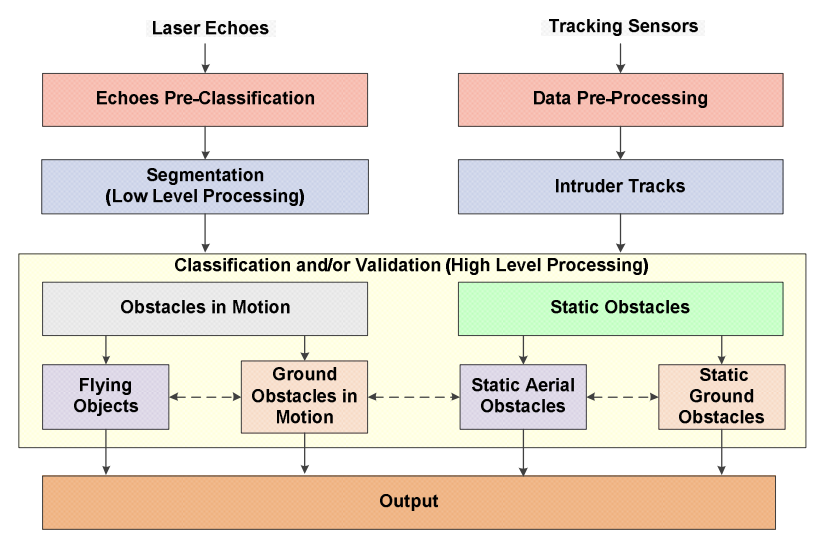

Fig. 4. LOWAS signal processing software architecture.

The LLP is performed on the individual echoes in order to determine range, angular coordinates and characteristics of the obstacle portion generating them. The tracking data processing provides the tracks of intruders after pre-processing (excluding pre-filtered false alarms). The HLP analyses the LLP output to identify groups of echoes, in order to reconstruct shape and type of the obstacle. LOWAS is capable of detecting and classifying ground/aerial static and moving obstacles. Ground static obstacles are automatically classified according to the following classes:

- Wire: all thin obstacles like wires and cables (e.g., telecommunication/power lines and cableways);

- Tree: vertical obstacles of reduced frontal dimensions (e.g., trees, poles and pylons);

- Structure: extended obstacles (e.g., bridges, buildings and hills).
The single echoes are processed as soon as they are acquired. The wire LLP algorithm processes only the echoes whose magnitude is weaker than pre-defined thresholds. Subsequently, the wire HLP algorithm is employed on the subset of acquired echoes in the current frame. Clusters are merged into a single obstacle by means of iterative image segmentation, specifically implemented to identify echoes characterised by a uniform range. A statistical algorithm subsequently validates the merged echoes by verifying if the obstacle is generated by real aligned echoes or by noise data. The processing algorithms for extended obstacles (trees and structures) are also divided in two different phases: echo analysis and segmentation. The echoes already classified as extended objects are processed by a dedicated validation algorithm, since many of these are not generated by obstacles (like, for example, the ground). The segmentation algorithm is responsible of detecting, merging and validating clusters of echoes acquired over relatively short time intervals and showing the geometric properties of an obstacle. The LOWAS performs automatic prioritisation of the detected obstacles based on the risk represented according to the relevant range and provides timely visual and aural warnings to the flight crew. The dedicated signal processing algorithms grant reliable detection performance, independent from the platform motion, allowing a reconstruction of the obstacle shape without using navigation data (stand-alone integration) in low-dynamics platforms flying pre-defined trajectories. The LOWAS can also be integrated with the navigation and guidance system of the UAV to grant more efficient and reliable obstacle detection in high-dynamics and no pre-planned flight conditions [12].

\subsection{History function}

A history function is implemented to retain obstacles information even when the previously observed obstacles are outside the current FOV of the LOWAS. Such a capability enables the storage of obstacle positions and other attributes for a certain period of time, which is automatically adjusted based on platform-obstacle relative dynamics. Since both obstacle data and platform navigation data are affected by errors, a propagation of uncertainty is performed to grant a 2-sigma confidence level to position data of host platform and tracking information of obstacles.

\section{Detection and atmospheric propagation models}

The microwave radar range equation also applies to laser systems and the power received by the detector is given by:

$$
\mathrm{P}_{\mathrm{R}}=\frac{\mathrm{P}_{\mathrm{T}} \mathrm{G}_{\mathrm{T}}}{4 \pi \mathrm{R}^{2}} \cdot \frac{\sigma}{4 \pi \mathrm{R}^{2}} \cdot \frac{\pi \mathrm{D}^{2}}{4} \cdot \tau_{\mathrm{atm}} \cdot \tau_{\mathrm{sys}}
$$

where $P_{T}$ is the transmitter power, $G_{T}$ is the transmitter antenna gain, $R$ is the range $[\mathrm{m}], \mathrm{D}$ is the aperture diameter $[\mathrm{m}], \tau_{\mathrm{atm}}$ is the atmospheric transmittance and $\tau_{\text {sys }}$ is the system transmission factor. With laser systems, the transmitter antenna gain is substituted by the aperture gain, expressed by the ratio of the steradian solid angle of the transmitter beam width $\alpha^{2}$ to that of the solid angle of a sphere as given by:

$$
\mathrm{G}_{\mathrm{T}}=\frac{4 \pi}{\alpha^{2}}
$$

In case of laser beam widths in the order of $1 \mathrm{mrad}$, the typical aperture gain at laser wavelengths $(\lambda)$ is about $70 \mathrm{~dB}$. In the far field, the transmitter beam width can also be expressed in terms of aperture illumination constant, $\mathrm{K}_{\mathrm{a}}$ as:

$$
\alpha=\mathrm{K}_{\mathrm{a}} \frac{\lambda}{\mathrm{D}}
$$


This is the author pre-publication version. This paper does not include the changes arising from the revision, formatting and publishing process. The final paper that should be used for referencing is:

S. Ramasamy, R. Sabatini, A. Gardi and J. Liu, "LIDAR Obstacle Warning and Avoidance System for Unmanned Aerial Vehicle Sense-andAvoid.” Aerospace Science and Technology (Elsevier), vol. 55, pages 344-358, 2016. DOI: 10.1016/j.ast.2016.05.020

Substituting for $\mathrm{G}_{\mathrm{T}}$ and $\alpha$ in equation (1), we obtain:

$$
\mathrm{P}_{\mathrm{R}}=\frac{\mathrm{P}_{\mathrm{T}}}{16 \mathrm{R}^{4}} \cdot \frac{\sigma}{\lambda^{2}} \cdot \frac{\mathrm{D}^{4}}{\mathrm{~K}_{\mathrm{a}}^{2}} \cdot \tau_{\mathrm{atm}} \cdot \tau_{\mathrm{sys}}
$$

At $\lambda=1.064 \mu \mathrm{m}$, a $10 \mathrm{~cm}$ aperture has a far-field distance of approximately $20 \mathrm{~km}$. As a result, it is not unusual to operate in the near-field of the optical systems and hence the range equation is modified to account for near-field operations. This near-field effect modifies the beam width such that:

$$
\alpha=\sqrt{\left(\frac{\mathrm{K}_{\mathrm{a}} \mathrm{D}}{R}\right)^{2}+\left(\frac{\mathrm{K}_{\mathrm{a}} \lambda}{\mathrm{D}}\right)^{2}}
$$

The range equation is dependent on the target area. The effective target cross-section is given by:

$$
\sigma=\frac{4 \pi}{\Omega} \rho \mathrm{dA}
$$

where $\Omega$ is the scattering solid angle of target [sr], $\rho$ is the target reflectivity and $\mathrm{dA}$ is the target area. Substituting $\Omega$ with the value associated with the standard scattering diffuse target (Lambertian target) having a solid angle of $\pi$ steradians, we obtain:

$$
\sigma=4 \rho_{\mathrm{T}} \mathrm{dA}
$$

The cross-sectional area of a laser beam transmitted by a circular aperture from a distance is given by:

$$
\mathrm{dA}=\frac{\pi \mathrm{R}^{2}}{4} \Theta^{2}
$$

Depending on the target-laser spot relative dimensions we may distinguish three different types of targets: extended, point and linear targets. In case of a point target (Fig. 5-a), the target crosssection is given by:

$$
\sigma_{\mathrm{pt}}=4 \rho_{\mathrm{T}} \mathrm{dA}
$$

Hence the range equation is expressed as:

$$
P_{R}=\frac{P_{T}}{4 R^{2}} \cdot \frac{d A \rho_{T}}{R^{2}} \cdot \frac{D^{4}}{K_{a}^{2} \lambda^{2}} \cdot \tau_{\text {atm }} \cdot \tau_{\text {sys }}
$$

In case of a linear target such as a wire (Fig. 5-b), it can have a length larger than the illuminated area but a smaller width (d). The target cross-section is given by:

$$
\sigma_{\text {wire }}=4 \rho_{\text {wire }} \mathrm{R} \theta \mathrm{d}
$$

Replacing with the beam width provided in Eq. (3), the range equation is expressed as:

$$
P_{R}=\frac{P_{T}}{4 R^{2}} \cdot \frac{d \rho_{\text {wire }}}{R} \cdot \frac{D^{3}}{K_{a} \lambda} \cdot \tau_{a t m} \cdot \tau_{\text {sys }}
$$

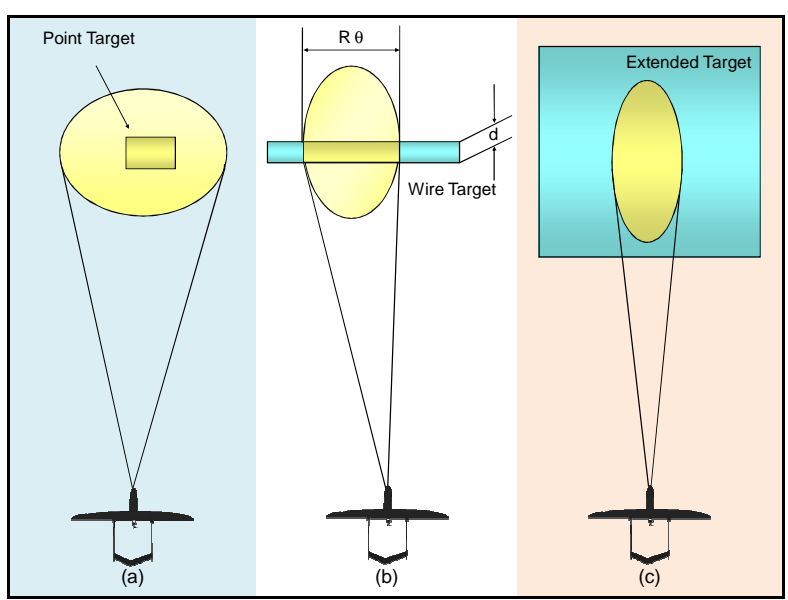

Fig. 5. Target cross sections.
In case of an extended target such as a wire (Fig. 5-c), all incident radiation is involved in the reflection process. Thus, for an extended Lambertian target we have a target cross-section given by:

$$
\sigma_{\text {ext }}=\pi \rho \mathrm{R}^{2} \theta^{2}
$$

Therefore the range equation is expressed as:

$$
\mathrm{P}_{\mathrm{R}}=\frac{\pi \mathrm{P}_{\mathrm{T}}}{4 \mathrm{R}} \cdot \frac{\mathrm{D}^{2} \rho}{4 \mathrm{R}} \cdot \tau_{\mathrm{atm}} \cdot \tau_{\text {sys }}
$$

The propagation of laser radiation in atmosphere is affected by a number of linear and nonlinear effects. Assuming a Gaussian profile of the laser beam at the source and an average focused irradiance, a comprehensive expression of the peak irradiance, $\mathrm{I}_{\mathrm{P}}$, accounting for absorption, scattering, diffraction, jitter, atmospheric turbulence and thermal blooming effects is given by $[11,13]$ :

$$
I_{P}(z, \lambda)=\frac{b(z) \tau(z, \lambda) P(\lambda)}{\pi\left(a_{d}^{2}(z, \lambda)+a_{j}^{2}(z)+a_{t}^{2}(z, \lambda)\right)}
$$

where $\mathrm{z}$ is linear coordinate along the beam, $\lambda$ is wavelength, $P(\lambda)$ is transmitted laser power, $b$ is blooming factor, $\tau(z, \lambda)$ is transmittance coefficient, which accounts for absorption and scattering associated with all molecular and aerosol species present in the path. The 1/e (e is Euler's number) beam radii associated with diffraction $\mathrm{a}_{\mathrm{d}}(\mathrm{z}, \lambda)$, beam jitter $\mathrm{a}_{\mathrm{j}}(\mathrm{z})$ and turbulence $\mathrm{a}_{\mathrm{t}}(\mathrm{z}, \lambda)$ are calculated as [14]:

$$
\begin{gathered}
a_{d}(z, \lambda)=\frac{Q z \lambda}{2 \pi a_{0}} \\
a_{j}^{2}(z)=2\left\langle\Theta_{\mathrm{x}}^{2}\right\rangle z^{2} \\
a_{t}(z, \lambda)=\frac{2 C_{N}^{6 / 5} z^{8 / 5}}{\lambda^{1 / 5}}
\end{gathered}
$$

where $Q$ is beam quality factor, $a_{0}$ is beam 1/e radius, $\left\langle\Theta_{x}^{2}\right\rangle$ is the variance of the single axis jitter angle that is assumed to be equal to $\left\langle\Theta_{\mathrm{y}}^{2}\right\rangle$ and $\mathrm{C}_{\mathrm{N}}^{2}$ is the refractive index structure constant. An empirical model for the blooming factor $b(z)$, which is the ratio of the bloomed $\mathrm{I}_{\mathrm{B}}$ to unbloomed $\mathrm{I}_{\mathrm{UB}}$ peak irradiance, is given by:

$$
\mathrm{b}(\mathrm{z})=\frac{\mathrm{I}_{\mathrm{B}}}{\mathrm{I}_{\mathrm{UB}}}=\frac{1}{1+0.0625 \mathrm{~N}^{2}(\mathrm{z})}
$$

where $\mathrm{N}$ is thermal distortion parameter and is a dimensionless quantity that indicates the degree or strength of thermal distortion given by:

$$
\mathrm{N}(\mathrm{z})=\frac{-\mathrm{n}_{\mathrm{T}} \alpha_{\mathrm{m}} \mathrm{Pz} \mathrm{z}^{2}}{\pi \mathrm{n}_{0} \mathrm{~d}_{0} \mathrm{v}_{0} \mathrm{c}_{\mathrm{P}} \mathrm{a}_{0}^{3}} \cdot\left[\frac{2}{\mathrm{z}^{2}} \int_{0}^{\mathrm{z}} \frac{\mathrm{a}_{0}}{\mathrm{a}\left(\mathrm{z}^{\prime}\right)} \mathrm{dz}^{\prime} \int_{0}^{\mathrm{z}^{\prime}} \frac{\mathrm{a}_{0}^{2} \mathrm{v}_{0} \tau \prime \prime}{\mathrm{a}} \mathrm{dz}^{\prime \prime}\right]
$$

where the multiplication factor is the distortion parameter for a collimated Gaussian beam of $1 / e$ radius $\mathrm{a}_{0} ; \mathrm{n}_{0}$ is refractive index; $\mathrm{v}_{0}$ is the uniform wind velocity in the weak attenuation limit $(\gamma \mathrm{z} \ll 1) ; \mathrm{P}$ is the laser output power; $\mathrm{n}_{\mathrm{T}}, \alpha_{\mathrm{m}}, \mathrm{d}_{0}$ and $\mathrm{c}_{\mathrm{P}}$ are, respectively, the coefficients of index change with respect to temperature, absorption coefficient, density and specific heat at constant pressure. The atmospheric transmittance $\tau$ depends on the integral effects of absorption and scattering phenomena, both for molecular and aerosol species, on the entire beam length, which are comprehensively described as:

$$
\tau(\mathrm{z}, \lambda)=\mathrm{e}^{-\int_{0}^{\mathrm{z}} \gamma(\mathrm{z}, \lambda) \mathrm{dz}}
$$

where $\gamma(\mathrm{z}, \lambda)$ is the atmospheric extinction. In the practical case, the molecular and aerosol composition of the atmosphere along the entire LIDAR beam is unknown and cannot be accurately guessed, therefore it is necessary to adopt an empirical model for the atmospheric extinction. Considering that the LIDAR 
S. Ramasamy, R. Sabatini, A. Gardi and J. Liu, "LIDAR Obstacle Warning and Avoidance System for Unmanned Aerial Vehicle Sense-andAvoid.” Aerospace Science and Technology (Elsevier), vol. 55, pages 344-358, 2016. DOI: 10.1016/j.ast.2016.05.020

operational wavelength is fixed and assuming that variations in the transmittance are caused by changes in the water content of the air only, $\gamma(\mathrm{z}, \lambda)$ can be calculated using the model suggested by Elder and Strong [15] and modified by Langer [16]. Additionally, for propagation in rainy conditions, the equations developed by Middleton can be adopted [17]. The Elder-StrongLanger-Middleton (ESLM) model relates the atmospheric transmission of the $i^{\text {th }}$ window to the atmospheric visibility, relative humidity and rainfall-rate (i.e., readily measurable parameters). This is a valid assumption since other atmospheric constituents have reasonably constant effects within the given atmospheric window. The number of $\mathrm{H}_{2} \mathrm{O}$ molecules encountered by the laser beam is expressed by the amount of precipitable water, which equals to the depth of the layer that would be formed if all the water molecules along the propagation path were condensed in a container having the same cross-sectional area as the beam. Hence, for a beam path length of $\mathrm{z}$ metres, the total precipitable water amount in millimetres is given by:

$$
\mathrm{w}=10^{-3} \rho \cdot \mathrm{z}
$$

where $\rho$ is the absolute humidity $\left[\mathrm{g} / \mathrm{m}^{3}\right]$. Two empirical expressions, developed by Langer can be used to calculate the absorptive transmittance $\tau_{\mathrm{ai}}$ for the $i^{\text {th }}$ window for any given value of the precipitable water content [14]. These transmittances are expressed as:

$$
\begin{gathered}
\tau_{a i}=e^{-A_{i} \sqrt{w}} \text { for } w<w_{i} \\
\tau_{a i}=k_{i}\left(\frac{w_{i}}{w}\right)^{\beta_{i}} \text { for } w>w_{i}
\end{gathered}
$$

where $A_{i}, k_{i}, \beta_{i}$ and $w_{i}$ are constants whose values for each atmospheric window are listed in [18]. For the LOWAS wavelength $\left(\lambda=1550 \mathrm{~nm}-4^{\text {th }}\right.$ atmospheric window $), A_{i}=0.211$, $\mathrm{k}_{\mathrm{i}}=0.802, \beta_{\mathrm{i}}=0.111$ and $\mathrm{w}_{\mathrm{i}}=1.1$. These empirical equations apply to horizontal paths in the atmosphere at sea-level and for varying relative humidity. To obtain the total atmospheric transmittance, $\tau_{\mathrm{ai}}$ is multiplied by $\tau_{\mathrm{si}}$ (i.e., the transmittance due to scattering only). Based on rigorous mathematical approaches, the scattering properties of the atmosphere due to the aerosol particles are difficult to quantify and it is difficult to obtain an analytic expression for the scattering coefficient that will yield accurate values over a wide variety of conditions. However, an empirical relationship that is often used to model the scattering coefficient has the form:

$$
\beta(\lambda)=C_{1} \lambda^{-\delta}+C_{2} \lambda^{-4}
$$

where $\mathrm{C}_{1}, \mathrm{C}_{2}$ and $\delta$ are constants determined by the aerosol concentration and size distribution and $\lambda$ is the wavelength of the radiation. The second term accounts for Rayleigh scattering, which may be neglected for all wavelengths longer than about 0.3 $\mu \mathrm{m}$. The values $\delta \approx 1.3 \pm 0.3$ produce reasonable results when applied to aerosols with a range of particle sizes. An attempt has also been made to relate $\delta$ and $\mathrm{C}_{1}$ to the meteorological range. The apparent contrast $\mathrm{C}_{\mathrm{Z}}$, of a source when viewed at $\lambda=0.55 \mu \mathrm{m}$ from a distance $\mathrm{z}$ is given by:

$$
\mathrm{C}_{\mathrm{Z}}=\frac{\mathrm{R}_{\mathrm{SZ}}+\mathrm{R}_{\mathrm{bZ}}}{\mathrm{R}_{\mathrm{bZ}}}
$$

where $R_{S Z}$ and $R_{b Z}$ are the apparent radiances of the source and its background as seen from a distance z. For $\lambda=0.55 \mu \mathrm{m}$, the distance at which the ratio:

$$
\mathrm{V}=\frac{\mathrm{C}_{\mathrm{Z}}}{\mathrm{C}_{0}}=\frac{\frac{\mathrm{R}_{\mathrm{SZ}}-\mathrm{R}_{\mathrm{bZ}}}{\mathrm{R}_{\mathrm{bZ}}}}{\frac{\mathrm{R}_{\mathrm{S} 0}-\mathrm{R}_{\mathrm{b} 0}}{\mathrm{R}_{\mathrm{b} 0}}}=0.02
$$

is defined as the meteorological range $\mathrm{V}$ (or visual range). It must be observed that this quantity is different from the observer visibility $\left(\mathrm{V}_{\text {obs }}\right)$. Observer visibility is the greatest distance at which it is just possible to see and identify a target with the unaided eye. The International Visibility Code (IVC) designations are too broad for scientific applications. If only an estimated observer visibility $\left(\mathrm{V}_{\mathrm{obs}}\right)$ is available, the meteorological range (V) are estimated from [11]:

$$
\mathrm{V} \approx(1.3 \pm 0.3) \cdot \mathrm{V}_{\mathrm{obs}}
$$

Assuming that the source radiance is much greater than the background radiance (i.e., $\mathrm{R}_{\mathrm{S}} \gg \mathrm{R}_{\mathrm{b}}$ ) and that the background radiance is constant (i.e., $\mathrm{R}_{\mathrm{b} 0}=\mathrm{R}_{\mathrm{bZ}}$ ), the transmittance at $\lambda=$ $0.55 \mu \mathrm{m}$ (where absorption is negligible) is given by:

$$
\frac{\mathrm{R}_{\mathrm{SV}}}{\mathrm{R}_{\mathrm{S} 0}}=\mathrm{e}^{-\beta \mathrm{V}}=0.02
$$

Hence:

$$
\ln \left(\frac{\mathrm{R}_{\mathrm{Sv}}}{\mathrm{R}_{\mathrm{S} 0}}\right)=-\beta \mathrm{V}=-3.91
$$

and also:

$$
\beta=\frac{-3.91}{\mathrm{~V}}=\mathrm{C}_{1} \lambda^{-\delta}
$$

The constant $\mathrm{C}_{1}$ is given by:

$$
\mathrm{C}_{1}=\frac{3.91}{\mathrm{~V}} \cdot 0.55^{\delta}
$$

The transmittance at the centre of the $i^{\text {th }}$ window is expressed as:

$$
\tau_{\mathrm{si}}=\mathrm{e}^{-\frac{3.91}{\mathrm{~V}} \cdot\left(\frac{\lambda_{\mathrm{i}}}{0.55}\right)^{-\delta} \cdot \mathrm{z}}
$$

where $\lambda_{i}$ is expressed in microns. If, because of haze, the meteorological range is less than $6 \mathrm{~km}$, the exponent $\delta$ is related to the meteorological range by the following empirical formula:

$$
\delta=0.585 \cdot \sqrt[3]{\mathrm{V}}
$$

where $\mathrm{V}$ is in kilometres. When $\mathrm{V} \geq 6 \mathrm{~km}$, the exponent $\delta$ can be calculated by:

$$
\delta=0.0057 \cdot \mathrm{V}+1.025
$$

For exceptionally good visibility, $\delta=1.6$ and for average visibility $\delta \approx 1.3$. In summary, an appropriate value for $\delta$ allows to compute the scattering transmittance at the centre of the $i^{\text {th }}$ window for any propagation path, if the meteorological range $\mathrm{V}$ is known. The extinction coefficients were computed from ESLM model transmittances using [2]:

$$
\gamma=-\frac{\ln \tau}{\mathrm{SR}}
$$

where SR is the slant range in $\mathrm{km}$. However, it is important to observe that, although the ESLM model provides independent estimates of both absorptive transmittance $\left(\tau_{a i}\right)$ and scattering transmittance $\left(\tau_{s i}\right)$, only the scattering contribution to the extinction coefficient $\left(\gamma_{\mathrm{si}}\right)$ is independent of range. It should be noted that, in fact, the total precipitable water in $\mathrm{mm}$ per $\mathrm{m}$ of beam length is given by Eq. (22) and absolute humidity is approximated by:

$$
\rho=1322.8 \frac{\mathrm{RH}}{\mathrm{T}} \exp \left[\frac{25.22(\mathrm{~T}-273.16)}{\mathrm{T}}-5.31 \ln \left(\frac{\mathrm{T}}{273.16}\right)\right]
$$

where $\mathrm{RH}$ is the relative humidity (as a fraction), and $\mathrm{T}$ is the absolute temperature $\left({ }^{\circ} \mathrm{K}\right)$. According to the ESLM model, the ESLM absorptive transmittance is given by [11]: 
This is the author pre-publication version. This paper does not include the changes arising from the revision, formatting and publishing process. The final paper that should be used for referencing is:

S. Ramasamy, R. Sabatini, A. Gardi and J. Liu, "LIDAR Obstacle Warning and Avoidance System for Unmanned Aerial Vehicle Sense-andAvoid.” Aerospace Science and Technology (Elsevier), vol. 55, pages 344-358, 2016. DOI: 10.1016/j.ast.2016.05.020

$$
\tau_{\mathrm{ai}}=\mathrm{e}^{-0.0363} \sqrt{w}
$$

Therefore, in this case, the absorptive extinction coefficient $\left(\gamma_{\mathrm{ai}} \mathrm{i}\right)$ is given by:

$$
\gamma_{\mathrm{ai}}=0.0363 \cdot \rho \cdot \frac{1}{\sqrt{\mathrm{SR}}}
$$

where the SR dependency of $\gamma_{\mathrm{ai}}$ is evident (obviously, for SR $=1$ $\mathrm{km}$ the model $\gamma_{\mathrm{ai}}$ becomes a function of AH only). The ESLM empirical model implies a range dependency of the extinction coefficient, which prevents direct comparisons of the experimental $\gamma$ values found at a certain SR with $\gamma$ values predicted or measured at a different SR.

\subsection{Detection range performance}

Extensive flight test activities performed on LOWAS for manned rotorcraft have addressed, in particular, the detection range performances in various weather and daylight conditions, as well as the Human-Machine Interface and Interaction $\left(\mathrm{HMI}^{2}\right)$ formats and functions and avoidance trajectory generation algorithms. The activities highlighted that the LOWAS range performances were in accordance with the predictions and the LOWAS detection/classification algorithms were validated. Furthermore, it was verified that the LOWAS history function was adequate to cover the flight envelope of the selected test platforms. Table 3 details the detection range results obtained for wire obstacles of 5 $\mathrm{mm}$ in diameter, in dry weather (visibilities of $800 \mathrm{~m}, 1500 \mathrm{~m}$ and $2000 \mathrm{~m}$ ) and incidence angles of $90^{\circ}$ and $45^{\circ}$. These results fulfil the minimum LOWAS performance requirements set for rotorcraft platforms. The detection ranges obtained by experiments also exceed the ESLM model detection ranges and this is due to a slight overestimation of the extinction coefficient at $\lambda=1.55 \mu \mathrm{m}$ as detailed in [2].

Table 3. Detection range of $5 \mathrm{~mm}$ diameter cable.

\begin{tabular}{|c|c|c|c|c|}
\hline $\begin{array}{c}\text { Visib- } \\
\text { ility }\end{array}$ & $\begin{array}{c}\text { Incid- } \\
\text { ence } \\
\text { Angle }\end{array}$ & $\begin{array}{c}\text { ESLM } \\
\text { Model } \\
\text { Detection } \\
\text { Distance }\end{array}$ & $\begin{array}{c}\text { Actual } \\
\text { Detection } \\
\text { Distance }\end{array}$ & $\begin{array}{c}\text { Minimum } \\
\text { Specified } \\
\text { Detection } \\
\text { Distance }\end{array}$ \\
\hline $800 \mathrm{~m}$ & $90^{\circ}$ & $662 \mathrm{~m}$ & $727 \mathrm{~m}$ & $500 \mathrm{~m}$ \\
\hline $1500 \mathrm{~m}$ & $90^{\circ}$ & $783 \mathrm{~m}$ & $832 \mathrm{~m}$ & $560 \mathrm{~m}$ \\
\hline $2000 \mathrm{~m}$ & $90^{\circ}$ & $921 \mathrm{~m}$ & $980 \mathrm{~m}$ & $600 \mathrm{~m}$ \\
\hline $800 \mathrm{~m}$ & $45^{\circ}$ & $495 \mathrm{~m}$ & $529 \mathrm{~m}$ & $400 \mathrm{~m}$ \\
\hline $1500 \mathrm{~m}$ & $45^{\circ}$ & $553 \mathrm{~m}$ & $623 \mathrm{~m}$ & $440 \mathrm{~m}$ \\
\hline $2000 \mathrm{~m}$ & $45^{\circ}$ & $629 \mathrm{~m}$ & $657 \mathrm{~m}$ & $520 \mathrm{~m}$ \\
\hline
\end{tabular}

\section{Human-machine interface and interactions}

Information relative to all detected obstacles is provided on dedicated avionics systems including 2D, 3D and altimetry display formats. Fig. 6 shows a visible image (a) and the corresponding LOWAS 3D display format (b). Fig. 6-c shows an enlarged version of the 3D display format. A 2D display format is shown in Fig. 6-d as well as a combined format is depicted in Fig. 6 -e, providing a synthetic augmented-reality image of the scene captured by visual camera and by LOWAS.

Comparing Fig. 6 (a) and (b), it is evident that pole and wire obstacles placed at a certain distance from the platform and hard to be seen by a visual camera (and by the human eye) are successfully detected and displayed by LOWAS. An altimetry display format is also available and depicted in Fig. 7. In all these avionics implementations, the actual platform orientation and flight path velocity vector with respect to the LOWAS axis are represented (an auto-alignment function can be also implemented if the required data are available from other avioncis navigation sensors).

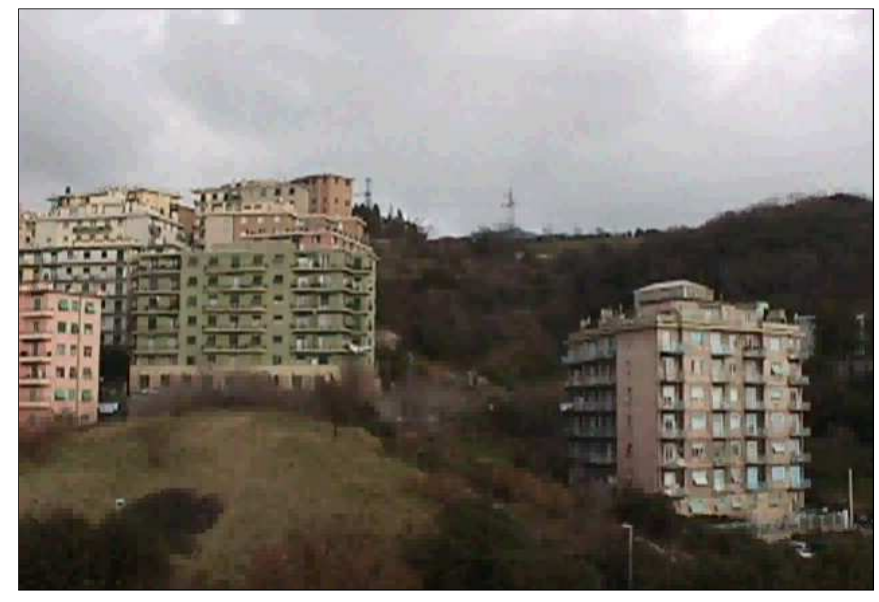

(a)

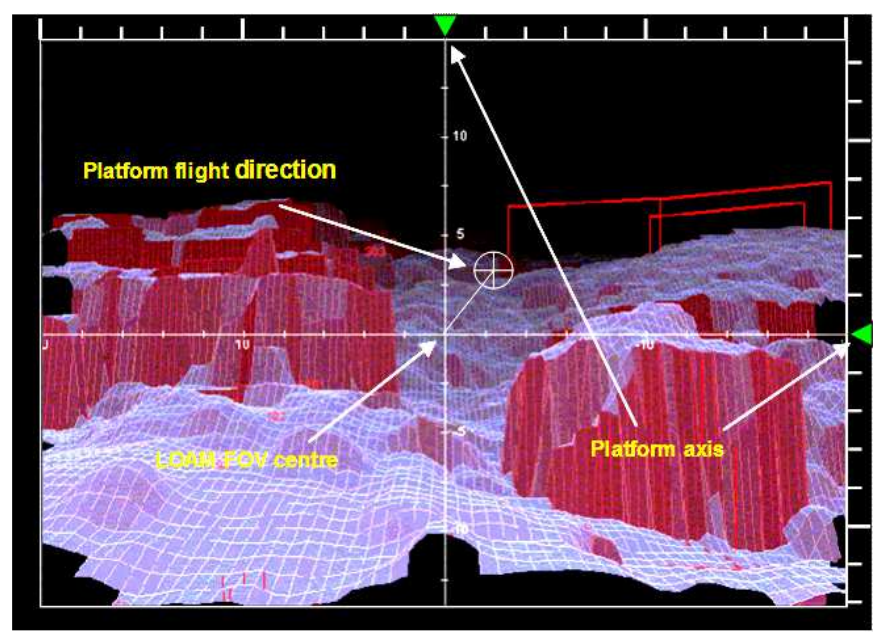

(b)

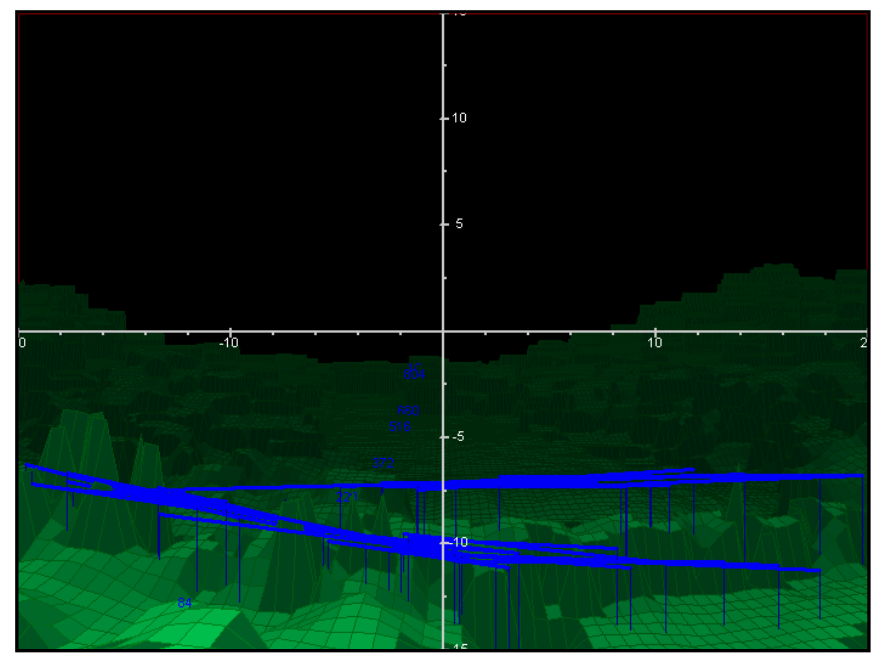

(c) 
This is the author pre-publication version. This paper does not include the changes arising from the revision, formatting and publishing process. The final paper that should be used for referencing is:

S. Ramasamy, R. Sabatini, A. Gardi and J. Liu, "LIDAR Obstacle Warning and Avoidance System for Unmanned Aerial Vehicle Sense-andAvoid.” Aerospace Science and Technology (Elsevier), vol. 55, pages 344-358, 2016. DOI: 10.1016/j.ast.2016.05.020

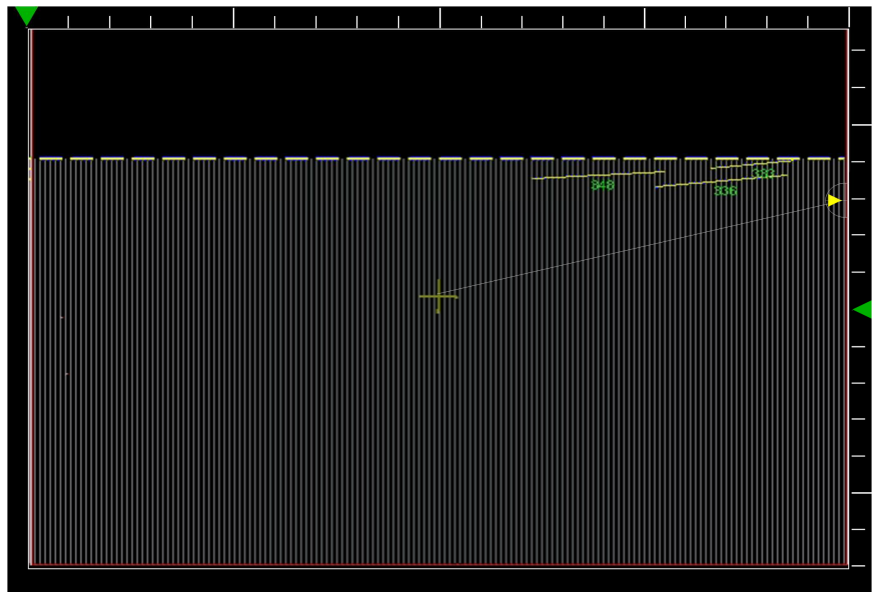

(d)

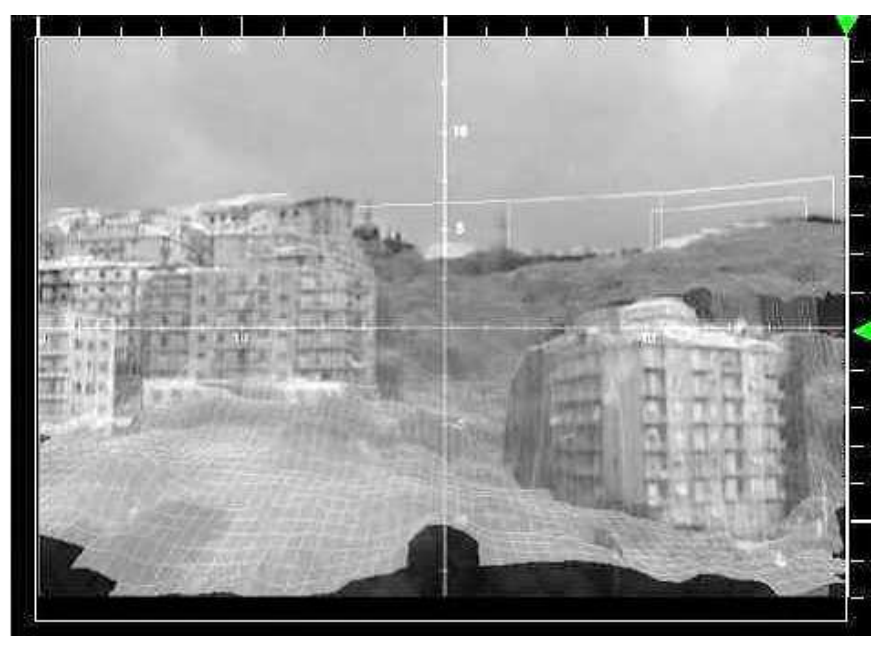

(e)

Fig. 6. Visible camera image (a), LOWAS 3D display (b and c) 2D display (d) and synthetic enhanced-reality image (e).

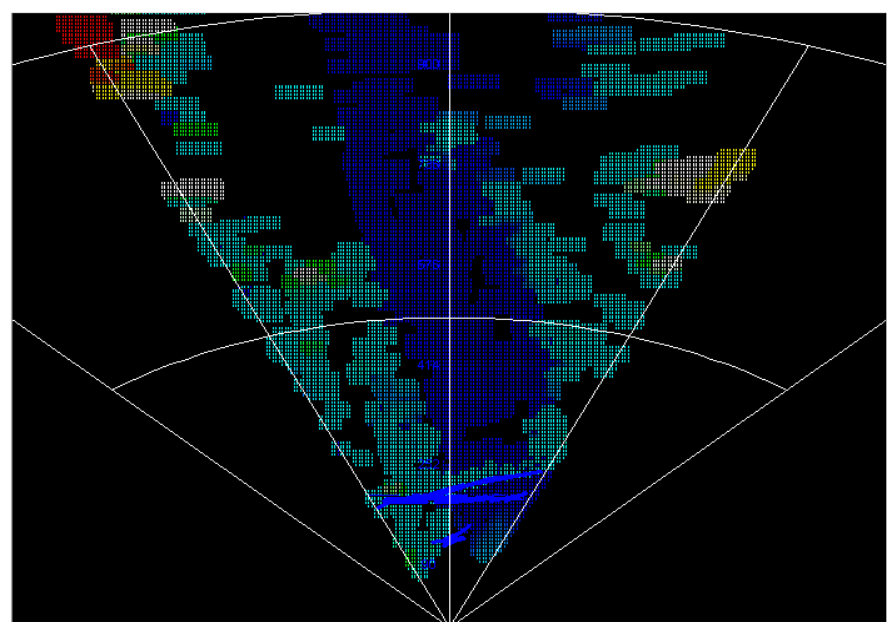

Fig. 7. LOWAS altimetry display formats.

\subsection{Cognitive remote pilot-UAV interface}

Cognitive ergonomics studies are performed to develop suitable $\mathrm{HMI}^{2}$ for LOWAS. Emphasis is on the development of adaptive forms of $\mathrm{HMI}^{2}$ based on the analysis of human cognitive states estimated from physiological measurements and external conditions. This information is used to trigger $\mathrm{HMI}^{2}$ adaptation (including various levels of automation) with the intention of increasing safety and efficiency of UAS low-level flight operations. In the LOWAS for UAS, a cognitive remote pilotaircraft interface is developed to dynamically assist remote pilots based on their physiological and cognitive states detected in realtime. Four intelligent and adaptive functions including real-time monitoring of environmental and operational statuses as well as pilot physiological parameters, adaptive alerting and dynamic task allocation are adopted to ensure an optimal cooperation between pilots and advanced aircraft systems [19-22].

Depending upon the current flight phase, environmental conditions, operational requirements and cognitive states of the remote pilot, suitably defined decision logics perform the automatic selection of formats and functions adaptively. The realtime cognitive state of the UAS remote pilot is estimated based on the detected physiological parameters (heart rate, respiration rate, blink rate, blood pressure, etc.). Several cognitive state parameters are considered for the design of adaptive $\mathrm{HMI}^{2}$ system, typically including mental workload, mental fatigue, vigilance, stress, etc. Normalised variables (i.e., defined between 0 and 1 and non-dimensional) are here adopted to express cognitive state parameters. Each of them has specific dependencies on physiological parameters of the remote pilot, which are expressed as:

$$
\psi_{\mathrm{j}, \mathrm{t}}{ }^{\prime}=\mathrm{f}\left(\varphi_{\mathrm{i}, \mathrm{t}}\right)
$$

where $\psi$ is a cognitive state parameter and $\varphi$ is a physiological parameter. As human physiological parameters are frequently dependent on age and gender, a reference cognitive state parameter $\left(\psi_{\mathrm{j}, \mathrm{ref}}{ }^{\prime}\right)$ is introduced as:

$$
\psi_{\mathrm{j}, \text { ref }}{ }^{\prime}=\mathrm{f}\left(\varphi_{\mathrm{i}, \text { ref }}\right)
$$

where the $\varphi_{\mathrm{i} \text {,ref }}$ is the reference of the $i^{\text {th }}$ physiological parameters. Adopting a weighted sum, the $j^{\text {th }}$ real-time pilot cognitive state parameter $\psi_{\mathrm{j}}^{\prime}$ is estimated as:

$$
\psi_{\mathrm{j}}^{\prime}=\sum_{\mathrm{i}=1}^{\mathrm{n}} \alpha_{\mathrm{i}} \cdot \frac{\psi_{\mathrm{j}, \mathrm{t}^{\prime}-\psi_{\mathrm{j}, \mathrm{ref}}}}{\psi_{\mathrm{j}, \mathrm{ref}^{\prime}}}
$$

where $\alpha_{i}$ represent the weights of cognitive indicators and $n$ is the number of cognitive indices. In addition to physiologicallyderived values $\psi_{\mathrm{j}}^{\prime}$, corresponding cognitive state estimates $\psi_{\mathrm{j}}{ }^{\prime \prime}$ are determined by processing external conditions characterising the mission, retrieved from the Flight Management System (FMS). In particular, both operational and environmental complexities are considered and this is expressed by:

$$
\psi_{\mathrm{j}}{ }^{\prime \prime}=\sum_{\mathrm{w}=1}^{\mathrm{p}} \alpha_{\mathrm{w}} \cdot \frac{\gamma_{\mathrm{w}, \mathrm{t}}}{\gamma_{\mathrm{w}, \mathrm{t}-1}}+\sum_{\mathrm{v}=1}^{\mathrm{q}} \alpha_{\mathrm{v}} \cdot \frac{\eta_{\mathrm{v}, \mathrm{t}}}{\eta_{\mathrm{v}, \mathrm{t}-1}}
$$

where $\alpha_{w}$ represents the scaling factor of the operational complexity $\left(\gamma_{\mathrm{w}}\right), \gamma_{\mathrm{w}, \mathrm{t}}$ is the real-time determined operational complexity, $\gamma_{\mathrm{w}, \mathrm{t}-1}$ is the operational complexity in the previous epoch; $\alpha_{v}$ represents the scaling factor of environmental complexity $\left(\eta_{v}\right), \eta_{v, t}$ is the real-time detected environmental complexity and $\eta_{\mathrm{v}, \mathrm{t}-1}$ is the environmental complexity detected in the previous epoch. The total cognitive state parameters are finally determined as a weighted-sum between the physiologically-derived values $\psi_{\mathrm{j}}^{\prime}$ and the estimates based on external conditions $\psi_{\mathrm{j}}^{\prime \prime}$ as:

$$
\psi_{\mathrm{j}}=\delta_{\mathrm{m}} \psi_{\mathrm{j}}^{\prime}+\delta_{\mathrm{e}} \psi_{\mathrm{j}}^{\prime \prime}
$$

where $\delta_{\mathrm{m}}$ is the weight associated to physiologically-derived values and $\delta_{\mathrm{e}}$ is the weight associated with estimates based on external conditions. The cognitive state parameters are processed 
This is the author pre-publication version. This paper does not include the changes arising from the revision, formatting and publishing process. The final paper that should be used for referencing is:

S. Ramasamy, R. Sabatini, A. Gardi and J. Liu, "LIDAR Obstacle Warning and Avoidance System for Unmanned Aerial Vehicle Sense-andAvoid.” Aerospace Science and Technology (Elsevier), vol. 55, pages 344-358, 2016. DOI: 10.1016/j.ast.2016.05.020

by suitably defined decision logics to trigger interface adaptation and hence are an essential constituent of the cognitive UAS remote pilot interface.

\subsection{Formats and functions}

The formats and functions are adaptively selected based on the following criteria:

- Safety Line: selected when the airspeed is low.

- Wires and Pole Obstacles (e.g., vertical pylons and bare trees): selected when wires and pole obstacles are within the OWS detection range.

- All Ground Obstacles: selected when wire/poles and other types of obstacles are within the OWS detection range.

- Air Obstacles: selected when aerial obstacles are within the OWS detection range.

- OWS 3D (colour-coded LIDAR image): selected when a superimposed Forward Looking Infrared (FLIR) image is required for low-light and night time operations.

In addition to the formats and functions listed above, various symbols are used to enhance the representation of detected obstacles and to assist in the decision making process:

- Distance from obstacle;

- Isolated obstacles (buildings, groups of trees, etc.);

- Integrity flags (cautions and warnings);

- Flight vector;

- Evade advice cue;

- Plan Position Indicator (PPI);

- Terrain map.

\subsection{LOWAS alerts}

Three general levels of alert are defined for LOWAS (i.e., warning, caution and advisory). These alerts are provided to the remote pilot through aural and visual outputs as shown in Table 4. The warning cues, cautions and advisory alerts are provided in the form of tone and digital voice outputs; and/or displayed on the system warning panel, Helmet-Mounted Sight/Display (HMS/D) and Multi-Function Display (MFD).

Table 4. LOWAS Alerts.

\begin{tabular}{|l|c|c|c|c|c|}
\hline & Tone & $\begin{array}{c}\text { Direct } \\
\text { Voice } \\
\text { O/P }\end{array}$ & $\begin{array}{c}\text { Warn- } \\
\text {-ing } \\
\text { Panel }\end{array}$ & $\begin{array}{c}\text { HM } \\
\text { S/D }\end{array}$ & MFD \\
\hline Warning cue & - & $\checkmark$ & - & $\checkmark$ & $\checkmark$ \\
\hline Caution & $\checkmark$ & - & $\checkmark$ & $\checkmark$ & $\checkmark$ \\
\hline Advisory & - & - & - & - & $\checkmark$ \\
\hline
\end{tabular}

The warning cues are triggered when a detected obstacle is within the selected range or 10" from the impact point. Cautions are produced when the obstacle is within the FOV or there occurs a failure/degradation of the OWS functions. Advisories are issued when obstacles are outside the FOV but within the UAV operational envelope (i.e., history function).

\section{SAA unified approach}

In order to develop a certifiable UAS SAA system consisting of suitable intruder detection equipment and data fusion algorithms, a combination of navigation and tracking sensors/systems is necessary. Global Navigation Satellite Systems (GNSS), Inertial Measurement Unit (IMU) and Vision Based Navigation (VBN) sensors are typically adopted in an UAV's navigation and guidance system architecture. Errors in the obstacle/intruder measurements are estimated considering a combination of noncooperative sensors, including active/passive Forward-Looking Sensors (FLS) and acoustic sensors, as well as cooperative systems, including Automatic Dependent Surveillance Broadcast (ADS-B) and Traffic Collision Avoidance System (TCAS). A SAA reference system architecture is shown in Fig. 8. The sequential steps involved in the SAA process for executing a failsafe Track, Decide and Avoid (TDA) loop are also depicted in Fig. 8.

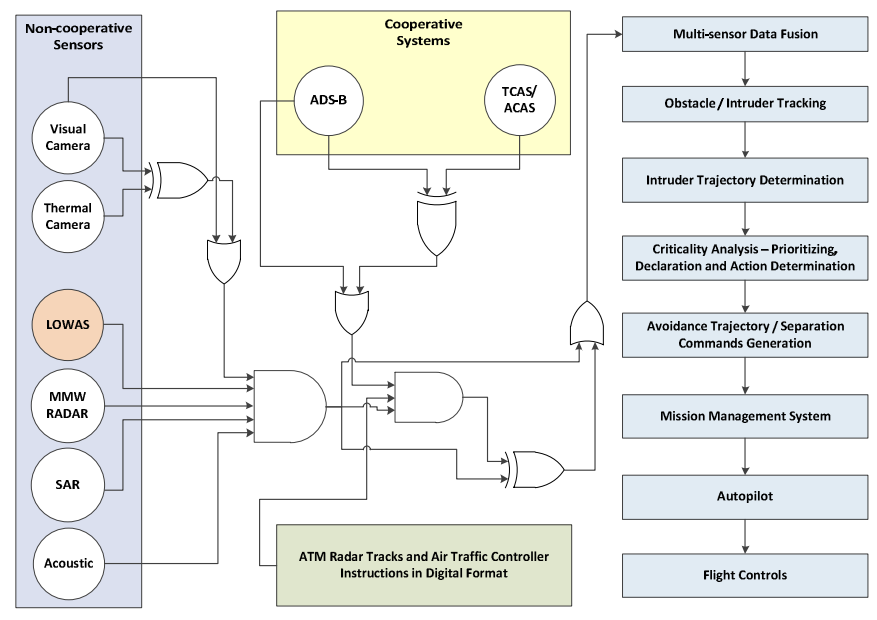

Fig. 8. SAA reference system architecture.

Non-cooperative sensors are employed to detect intruders or other obstacles in the UAV Field of Regard (FOR) when cooperative systems are unavailable to the intruders. Optical, thermal, LOWAS, Millimetre Wave (MMW) radar and acoustic sensors are employed as non-cooperative sensors. Currently, the inclusion of ADS-B redefines the paradigm of Communication, Navigation and Surveillance (CNS) in ATM by providing trajectory information in case of cooperative systems in addition to the use of Traffic Collision Avoidance System (TCAS)/Airborne Collision Avoidance System (ACAS). Boolean decision logics are applied for optimal selection and fusion of data obtained from state-of-the-art non-cooperative sensors, cooperative systems and ATM radar tracks/air traffic controller instructions in digital format transmitted by data links. The trajectory information of the intruders is determined after performing multi-sensor data fusion. Criticality analysis is carried out to prioritise (i.e., to determine if the specified collision risk threshold is exceeded by the tracked intruders) and to determine the action commands. In order to estimate the overall avoidance volume, navigation error of the host platform and tracking error of obstacles/intruders are evaluated and combined. The variation in the state vector (host and intruder) is expressed as:

$$
\delta\left(\mathrm{X}_{\mathrm{i}}(\mathrm{t})\right)=\left[\frac{\delta \mathrm{X}}{\delta \mathrm{p}}\right]_{\mathrm{t}} \cdot \sigma_{\mathrm{p}_{\mathrm{j}}}
$$

where $p$ is the position of the UAV and $t$ is the time of measurement. Let $\mathrm{R}, \alpha$ and $\epsilon$ be the range, azimuth and elevation obtained from LOWAS. Let $\mathrm{R}_{0}, \alpha_{0}$ and $\epsilon_{0}$ be the nominal range, 
This is the author pre-publication version. This paper does not include the changes arising from the revision, formatting and publishing process. The final paper that should be used for referencing is:

S. Ramasamy, R. Sabatini, A. Gardi and J. Liu, "LIDAR Obstacle Warning and Avoidance System for Unmanned Aerial Vehicle Sense-andAvoid.” Aerospace Science and Technology (Elsevier), vol. 55, pages 344-358, 2016. DOI: 10.1016/j.ast.2016.05.020

azimuth and elevation values. Let $\sigma_{\mathrm{R}}, \sigma_{\alpha}$ and $\sigma_{\epsilon}$ be the standard deviations of the errors in range, azimuth and elevation respectively. The associated error ellipsoids are given by:

$$
\frac{\left(\mathrm{R}-\mathrm{R}_{0}\right)^{2}}{\sigma_{\mathrm{R}}{ }^{2}}+\frac{\left(\alpha-\alpha_{0}\right)^{2}}{\sigma_{\alpha}^{2}}+\frac{\left(\epsilon-\epsilon_{0}\right)^{2}}{\sigma_{\epsilon}{ }^{2}}=1
$$

In the case of a static non-cooperative obstacle, the errors in range, azimuth and elevation are given by:

$$
\begin{gathered}
\delta \mathrm{R}=\mathrm{R}_{0}+\sigma_{\mathrm{R}} \cdot \sin \psi \\
\delta \alpha=\alpha_{0}+\sigma_{\alpha} \cdot \cos \varphi \cdot \cos \psi \\
\delta \epsilon=\epsilon_{0}+\sigma_{\epsilon} \cdot \sin \varphi \cdot \cos \psi
\end{gathered}
$$

where $\mathrm{R}_{0}, \alpha_{0}, \epsilon_{0}$ are the nominal range, azimuth and elevation measurements and $\{\varphi, \psi\}$ are parameterisation factors. The transformation of $\{\mathrm{R}, \alpha, \epsilon\}$ to $\{\mathrm{x}, \mathrm{y}, \mathrm{z}\}$ is given by:

$$
\begin{gathered}
\mathrm{x}=\mathrm{R} \cdot \cos \alpha \cdot \cos \epsilon \\
\mathrm{y}=\mathrm{R} \cdot \sin \alpha \cdot \cos \epsilon \\
\mathrm{z}=\mathrm{R} \cdot \sin \epsilon
\end{gathered}
$$

An example of the two combined navigation and tracking error ellipsoids assuming range only errors and the resulting uncertainty volume for uncorrelated and correlated (covariant and contravariant) sensor error measurements ( 3 out of a 27 total possibilities) is illustrated in Fig. 9. In the case of dynamic obstacles, the uncertainty volume is obtained based on a confidence region given by:

$$
\begin{gathered}
\delta \mathrm{v}_{0}=\mathrm{v}_{0}+\sigma_{\mathrm{v}_{0}} \cdot \sin \psi \\
\delta v_{0}=v_{0}+\sigma_{v_{0}} \cdot \cos \varphi \cdot \cos \psi \\
\delta v_{0}=v_{0}+\sigma_{v} \cdot \sin \varphi \cdot \cos \psi
\end{gathered}
$$

where $\mathrm{v}_{0}, v_{0}, \mathrm{v}_{0}$ are the nominal velocity measurements. When an error in elevation and azimuth is present, a conical inflation is obtained at the estimated range.

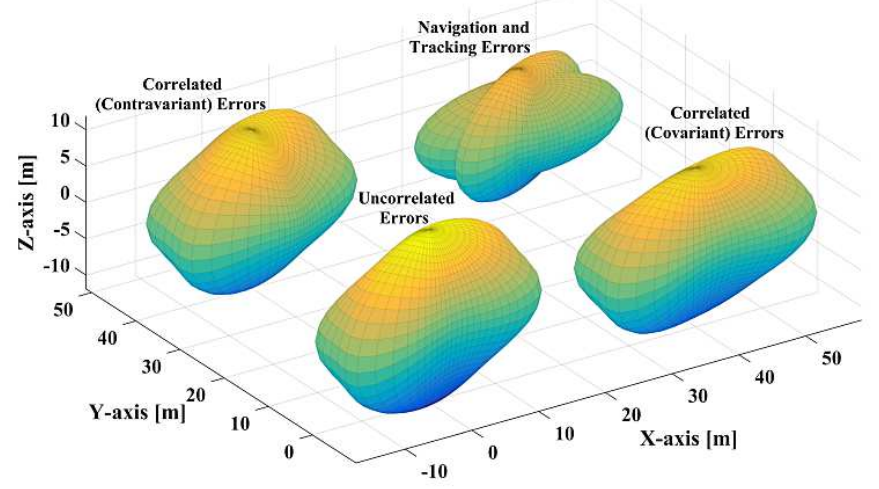

Fig. 9. Uncertainty volumes obtained from range only errors.

The kinematic relationships are:

$$
\begin{gathered}
\mathrm{v}_{\mathrm{x}}=\mathrm{v} \cdot \cos \mathrm{v} \cdot \cos \mathrm{u} \\
\mathrm{v}_{\mathrm{y}}=\mathrm{v} \cdot \sin \mathrm{v} \cdot \cos \mathrm{u} \\
\mathrm{v}_{\mathrm{z}}=\mathrm{v} \cdot \sin \mathrm{u}
\end{gathered}
$$

and these equations are governed according to the following laws of motion:

$$
\begin{aligned}
& x=x_{0}+v_{x} \cdot t \\
& y=y_{0}+v_{y} \cdot t \\
& z=z_{0}+v_{z} \cdot t
\end{aligned}
$$

The errors in $\{\mathrm{x}, \mathrm{y}, \mathrm{z}\}$ are given by:

$$
\begin{aligned}
& \sigma_{\mathrm{x}}^{2}=\left(\sigma_{\mathrm{x} 0}^{2}+\sigma_{\mathrm{v}_{\mathrm{x}} \cdot \mathrm{t}}{ }^{2}+2 \sigma_{\mathrm{x} 0 \mathrm{v}_{\mathrm{x}} \cdot \mathrm{t}}\right) \\
& \sigma_{\mathrm{y}}{ }^{2}=\left(\sigma_{\mathrm{y} 0}{ }^{2}+\sigma_{\mathrm{v}_{\mathrm{y}} \cdot \mathrm{t}}{ }^{2}+2 \sigma_{\mathrm{y} 0 \mathrm{v}_{\mathrm{y}} \cdot \mathrm{t}}\right) \\
& \sigma_{\mathrm{z}}{ }^{2}=\left(\sigma_{\mathrm{z} 0}{ }^{2}+\sigma_{\mathrm{v}_{\mathrm{z}} \cdot \mathrm{t}}{ }^{2}+2 \sigma_{\mathrm{z} 0 \mathrm{v}_{\mathrm{z}} \cdot \mathrm{t}}\right)
\end{aligned}
$$

The resultant avoidance volume obtained at an estimated range is shown in Fig. 10. A conceptual representation of the overall avoidance volume obtained in relation to an identified collision threat and the avoidance trajectory generated by the SAA system is provided in Fig. 11.

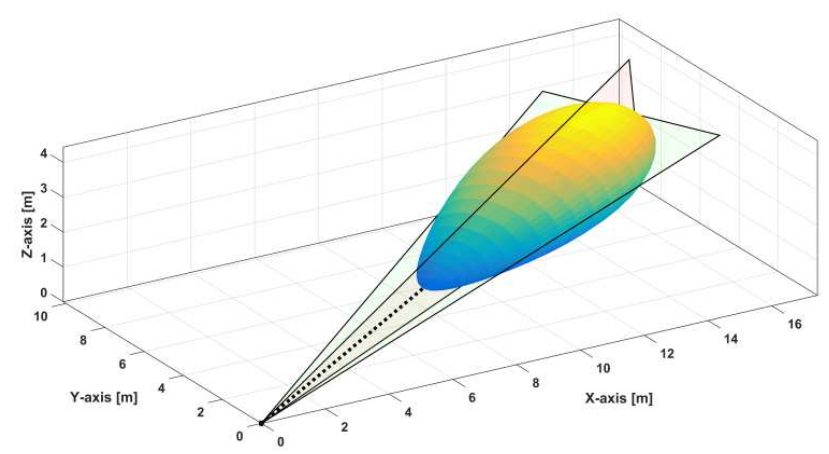

Fig. 10. Uncertainty volume at an estimated range.

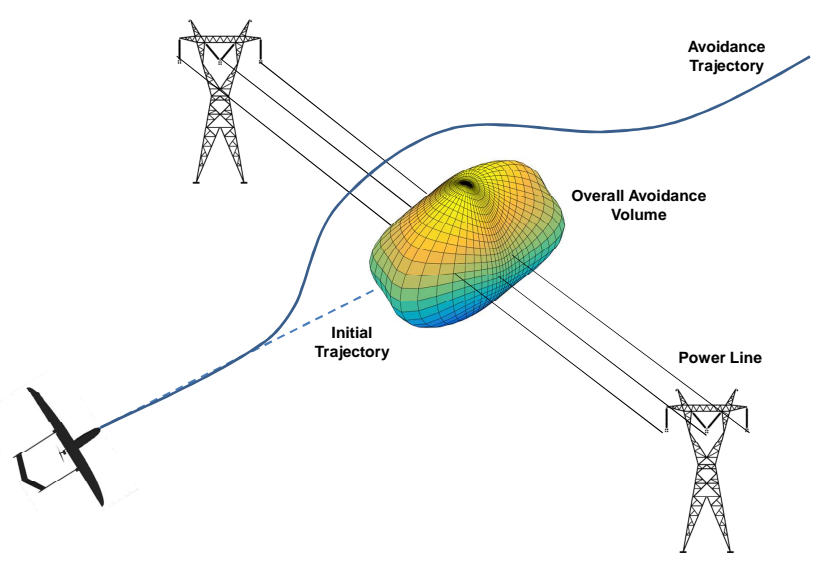

Fig. 11. Conceptual representation of the obstacle avoidance scenario.

In order to assure adequate safety levels, a separation buffer is introduced, which inflates the avoidance volume associated with each obstacle. In particular, to provide a 2-sigma confidence level, the uncertainty associated with the position of an obstacle is calculated as twice the standard deviation of the combined navigation and tracking error. When the distance between two detected obstacles is comparable with the calculated uncertainty values, or with the aircraft dimensions, the algorithm combines the two obstacles into a single overall avoidance volume. The key advantage is that the safe avoidance is determined by evaluating the risk-of-collision and then a safe manoeuvring point is identified from where the host UAV can manoeuvre safely (i.e., any manoeuvre can be performed within the UAV operational flight envelope). The risk of collision is evaluated by setting a 
S. Ramasamy, R. Sabatini, A. Gardi and J. Liu, "LIDAR Obstacle Warning and Avoidance System for Unmanned Aerial Vehicle Sense-andAvoid.” Aerospace Science and Technology (Elsevier), vol. 55, pages 344-358, 2016. DOI: 10.1016/j.ast.2016.05.020

threshold on the probability density function of a near mid-air collision event over the separation volume. The risk-of-collision is zero at the safe manoeuvring point.

\section{Generation of avoidance trajectories}

Once the obstacle has been detected, classified and prioritised as described in section 4 and the corresponding avoidance volume is computed in real-time as described in section 7 , the LOWAS triggers the generation of feasible avoidance trajectories based on platform dynamics and avoidance volumes. An optimal avoidance trajectory is subsequently identified among the feasible set based on robust multi-criteria decision logic. The adoption of trajectory optimisation algorithms in SAA represents a substantial evolution from the conventional safe-steering methodologies adopted in current systems [23]. In particular, in the context of conflict identification and resolution, trajectory optimisation allows the identification of the safest and more efficient Three-Dimensional or Four-Dimensional (3D/4D) avoidance trajectory, considering dynamics/airspace constraints, obstacle characteristics or intruder dynamics, as well as meteorological and traffic conditions. Current R\&D efforts are addressing practical implementations of advanced multi-model and multi-objective 3D/4D trajectory optimisation algorithms in novel ground-based and airborne Communication, Navigation and Surveillance/Air Traffic Management and Avionics (CNS+A) systems [22]. Most computationally efficient trajectory optimisation algorithms currently adopted in the aerospace domain belong to the family of direct methods. These solution methods involve the transcription of the infinite-dimensional trajectory optimisation problem formulated as optimal-control problem in a finite-dimensional Non-Linear Programming (NLP) problem, hence following the approach summarised as "discretise then optimise" [22-27]. This transcription problem can be either performed by introducing a control parameterisation based on arbitrarily chosen analytical functions, as in transcription methods, or by adopting a generalised piecewise approximation of both control and state variables based on a polynomial sequence of arbitrary degree, as in collocation methods. In both cases, the transcribed dynamical system is integrated along the time interval $\left[\mathrm{t}_{0} ; \mathrm{t}_{\mathrm{f}}\right]$. The search of the optimal set of discretisation parameters is formulated as a NLP problem, which is solved computationally by exploiting efficient numerical NLP algorithms. In direct transcription methods, a basis of known linearly independent functions $\mathrm{q}_{\mathrm{k}}(\mathrm{t})$ with unknown coefficients $\mathrm{a}_{\mathrm{k}}$ is adopted as the parameterisation in the general form:

$$
\mathrm{z}(\mathrm{t})=\sum_{\mathrm{k}=1}^{\mathrm{N}} \mathrm{a}_{\mathrm{k}} \mathrm{q}_{\mathrm{k}}(\mathrm{t})
$$

In direct shooting and multiple direct shooting methods, the parameterisation is performed on the controls $\mathrm{u}(\mathrm{t})$ only, the dynamic constraints are integrated with traditional numerical methods starting from the initial conditions and the Lagrange term in the cost function is approximated by a quadrature approximation. In the multiple shooting methods, the analysed time interval is partitioned into $n_{i}+1$ subintervals. The direct shooting method is then implemented at each subinterval. Parallel implementations of direct shooting involve the simultaneous integration of a family of trajectories based on different control parametrisation profiles, taking advantage of increasingly common multi-thread/multi-core hardware architectures. The optimal solution is determined a posteriori, both in the case of single objective and multi objective implementations.

Safety-critical applications of trajectory optimisation algorithms are actively investigated for airborne emergency Decision
Support Systems (DSS), also known as safety-nets, such as the LOWAS. These safety-critical CNS+A applications impose realtime requirements on the trajectory generation algorithm. Additionally, all generated trajectories must necessarily fulfil each and every constraint that is set, as the obstacle avoidance and manoeuvring envelope are formulated as constraints. These requirements restrict the choice of solution methods and multiobjective optimality decision logics that can be employed. In particular, a number of solution methods involve the intentional violation of constraints to promote convergence to optimality and among them the most conventional algorithms implemented are of collocation methods. Robust parallelised direct shooting solution methods with a posteriori decision logics are implemented for the generation of safe obstacle avoidance trajectories in the case of LOWAS for manned and unmanned aircraft. Since an optimal control-based formulation is adopted, the algorithm is based on the aircraft dynamics and not on a geometric trajectory models. The algorithm also accounts for path and airspace constraints as well as local meteorological conditions.

\subsection{Dynamics constraints}

The approximated dynamics model of the fixed-wing aircraft implemented as dynamics constraint in the LOWAS avoidance trajectory generation algorithm is based on the following assumptions:

- The aircraft is modelled as a point-mass with 3 linear Degrees-of-Freedom (3-DoF);

- The mass of the aircraft is considered constant along the avoidance trajectory;

- The inertial reference system adopted is fixed on the initial aircraft position (i.e. at the instant of detection of the first obstacle), with the $\mathrm{X}$-axis tangent to the original trajectory, the $\mathrm{Y}$-axis perpendicular to the trajectory and parallel to the ground and the Z-axis normal to the ground;

- The aircraft is subject to a constant gravitational acceleration parallel and opposite to the $\mathrm{Z}$ axis and for the current implementation a value of $g=9.81 \mathrm{~m} / \mathrm{s}^{2}$ is considered;

- The airspeed is expressed as True Air Speed (TAS). The assumed initial TAS is $\mathrm{v}=25 \mathrm{~m} / \mathrm{s}$. The effects of wind are considered in the dynamics model, but not simulated.

The resulting system of differential equations for 3 Degrees-ofFreedom (3-DoF) flight dynamics is:

$$
\left\{\begin{array}{l}
\dot{v}=\frac{\mathrm{T}-\mathrm{D}}{\mathrm{m}}-\mathrm{g} \sin \gamma \\
\dot{\gamma}=\frac{\mathrm{g}}{\mathrm{v}} \cdot(\mathrm{N} \cos \mu-\cos \gamma) \\
\dot{\chi}=\frac{\mathrm{g}}{\mathrm{v}} \cdot \frac{\mathrm{N} \sin \mu}{\cos \gamma} \\
\dot{x}=v \cos \gamma \sin \chi+v_{w_{x}} \\
\dot{y}=v \cos \gamma \cos \chi+v_{w_{y}} \\
\dot{z}=v \sin \gamma+\mathrm{v}_{\mathrm{w}_{\mathrm{z}}} \\
\dot{m}=-\mathrm{F}_{f}
\end{array}\right.
$$

where $\mathrm{D}$ is the aerodynamic drag $[\mathrm{N}], \mathrm{v}_{\mathrm{w}}$ is the wind velocity in its three scalar components $[\mathrm{m} / \mathrm{s}]$ and $\mathrm{F}_{f}=0$ is fuel flow $\left[\mathrm{kg} \mathrm{s}{ }^{-1}\right]$. The state vector consists of the following variables: aircraft mass $\mathrm{m}[\mathrm{kg}] ; \gamma$ is flight path angle [rad]; $\chi$ is track angle $[\mathrm{rad}] ; \mathrm{x}, \mathrm{y}, \mathrm{z}$ coordinates $[\mathrm{m}]$. The control variables are: load factor $\mathrm{N}$ [ ]; thrust force $\mathrm{T}[\mathrm{N}]$, bank angle $\mu$ [rad]. The accuracy of 3- 
This is the author pre-publication version. This paper does not include the changes arising from the revision, formatting and publishing process. The final paper that should be used for referencing is:

S. Ramasamy, R. Sabatini, A. Gardi and J. Liu, "LIDAR Obstacle Warning and Avoidance System for Unmanned Aerial Vehicle Sense-andAvoid.” Aerospace Science and Technology (Elsevier), vol. 55, pages 344-358, 2016. DOI: 10.1016/j.ast.2016.05.020

DoF flight dynamics is shown here to be adequate for lowdynamics platforms and in combination with smooth control logics leads to the generation of relatively smooth avoidance trajectories. Additional trajectory generation algorithms based on 6 Degrees-of-Freedom (6-DoF) dynamics are currently being developed, with aerodynamic and inertia coefficients retrieved as given in [28]. The same flight dynamics model is used both for the host platform and the intruder if the obstacle is a flying object.

\subsection{Path constraints}

The avoidance volumes generated as described in section 7 and parameterised with spherical harmonics as described in [23] are introduced as path constraint on the 3 spatial coordinates. The violation of the avoidance volumes leads the algorithm to interrupt the integration and discard the trajectory from the feasible set. During the avoidance manoeuvre, the load factor is set close to the certified flight envelope limits of the aircraft. The values correspond to $\mathrm{N}=\mathrm{N}_{\mathrm{MAX}}=2.5$ for pull-up manoeuvres and $\mathrm{N}=\mathrm{N}_{\mathrm{MIN}}=-1$ for diving manoeuvres. The limits on the flight path angle are set to $-60^{\circ}<\gamma<75^{\circ}$, whereas the limits on the bank angle are set to $-60^{\circ}<\mu<60^{\circ}$. During the entire approach to the obstacle, the vehicle control system is assumed to provide a linear variation of $\mu$ and $\mathrm{N}$ from the respective initial condition values, up to the assumed maximum values. The maximum roll rate adopted is $\dot{\mu}_{\mathrm{MAX}}=20 \%$ s.

\subsection{Multi-objective decision logics}

The subsequent step involves the selection of the optimal trajectory from the generated set of safe trajectories, which is then provided in the form of steering commands to the aircraft guidance subsystem. The implemented decision logics are based on minimisation of the following cost function:

$$
\mathrm{J}=\mathrm{w}_{\mathrm{t}} \cdot \mathrm{t}_{\mathrm{SAFE}}-\mathrm{w}_{\mathrm{d}} \cdot \mathrm{d}_{\mathrm{m}}(\mathrm{t})+\int\left[w_{f} \cdot \mathrm{SFC} \cdot \mathrm{T}(\mathrm{t})\right] \mathrm{dt}
$$

where, given $T_{T}$ as the time-to-threat and $T_{M}$ as the avoidance manoeuvre time, $t_{\mathrm{SAFE}}$ is the time at which the safe avoidance condition is successfully attained, defined as:

$$
\mathrm{t}_{\mathrm{SAFE}}=\mathrm{T}_{\mathrm{T}}+2 \mathrm{~T}_{\mathrm{A}}
$$

SFC $\left[\frac{\mathrm{kg}}{\mathrm{N}} \cdot \mathrm{s}\right]$ is specific fuel consumption, $\mathrm{T}(\mathrm{t})$ is thrust profile and the coefficients $w_{t}, w_{f}, w_{d}$ are the weights attributed to time, fuel and distance respectively. The term $d_{m}(t)$ corresponds to the minimum distance from the dynamic geo-fence, which is given by:

$$
\mathrm{d}_{m}(\mathrm{t})=\min \left[\sqrt{\left(\mathrm{x}(\mathrm{t})-\mathrm{x}_{\mathrm{GF}}(\mathrm{t})\right)^{2}+\left(\mathrm{y}(\mathrm{t})-\mathrm{y}_{\mathrm{GF}}(\mathrm{t})\right)^{2}+\left(\mathrm{z}(\mathrm{t})-\mathrm{z}_{\mathrm{GF}}(\mathrm{t})\right)^{2}}\right]
$$

where $\mathrm{x}_{\mathrm{GF}}, \mathrm{y}_{\mathrm{GF}}$ and $\mathrm{z}_{\mathrm{GF}}$ are the coordinates of the bounding surfaces of the dynamic geo-fence. Only the distances along $\mathrm{x}$ and $\mathrm{z}$ are considered for the wire obstacle, and the geo-fence is tangent to the obstacle avoidance volume computed according to the models provided in Section 7. This cost function is specifically designed for time-critical avoidance applications (i.e., closing-up obstacles with high relative velocities and/or accelerations). For a practical implementation, appropriate higher weightings are used for time and distance cost elements. Other cost elements with appropriate weightings can also be introduced as required. Depending on the relationship between the available time-to-collision and the computation time, pseudospectral optimisation and differential geometry optimisation methods may also be employed in less critical situations and the optimised avoidance trajectory data could be exchanged with the ground pilot and/or ground-based ATM systems for validation and execution. After computing the optimal avoidance trajectory up to a safe manoeuvring point, the SAA trajectory planning algorithm implements pseudospectral optimisation methods targeting minimum time and minimum fuel to re-join the original trajectory.

\section{Simulation case study}

Simulation case studies are performed in a realistic scenario to assess the LOWAS avoidance trajectory generation algorithms. The simulation scenario considers an UAV equipped with LOWAS flying towards a number of obstacles of different geometric characteristics as illustrated in Fig. 12. The UAV is flying at an altitude $\mathrm{z}=100 \mathrm{~m}$ Above Ground Level (AGL), at a relatively low speed $(20 \mathrm{~m} / \mathrm{s})$ and approaching a power transmission line and a tower building (the power line is composed of several wires $10 \mathrm{~mm}$ in diameter). The altitude of the lowest wire is $90 \mathrm{~m}$ AGL and the altitude of the highest wire is $110 \mathrm{~m} \mathrm{AGL}$; the wires are separated by about $6.5 \mathrm{~m}$ vertically and $5 \mathrm{~m}$ laterally. The power lines lie approximately $65 \mathrm{~m}$ in front of the UAV. The original horizontal flight trajectory would lead to a collision with the power line. After a successful detection of all wires, the algorithm calculates the distances to each of them. As described in section 7 , the algorithm recognises that the calculated distances are all comparable with the UAV size and therefore combines all wires in a single avoidance volume. As the obstacle is classified as ground-based obstacle of "wire" type, a dynamic geo-fence is also generated to ensure that even the extent of the wire beyond the LOWAS FOV is considered, so that no unsafe avoidance trajectory is generated. In particular, as the length of the wire obstacles is assumed to exceed the LOWAS FOV, this geo-fence has unlimited width. The building behind the transmission line is $155 \mathrm{~m}$ high, $50 \mathrm{~m}$ in length, $50 \mathrm{~m}$ in width, and located at $160 \mathrm{~m}$ in front of the UAV initial position. An additional avoidance volume is therefore computed for the building considered in the presented scenario, and a corresponding narrower (but taller) geo-fence is generated. After processing the information for all freshly detected obstacles, the trajectory avoidance algorithm calculates whether the original flight trajectory leads to a collision. When this condition occurs, a number of feasible avoidance trajectories are generated by the trajectory generation algorithm, among which the optimal flight path is selected. Based on the cost function presented in Section 8.3 , an optimal solution is obtained and the corresponding optimal avoidance trajectory is selected as depicted in Fig. 13. In this case, the only non-zero weight in the cost functional of Eq. (67) is the one associated to the integral distance, $\mathrm{w}_{\mathrm{d}}$.

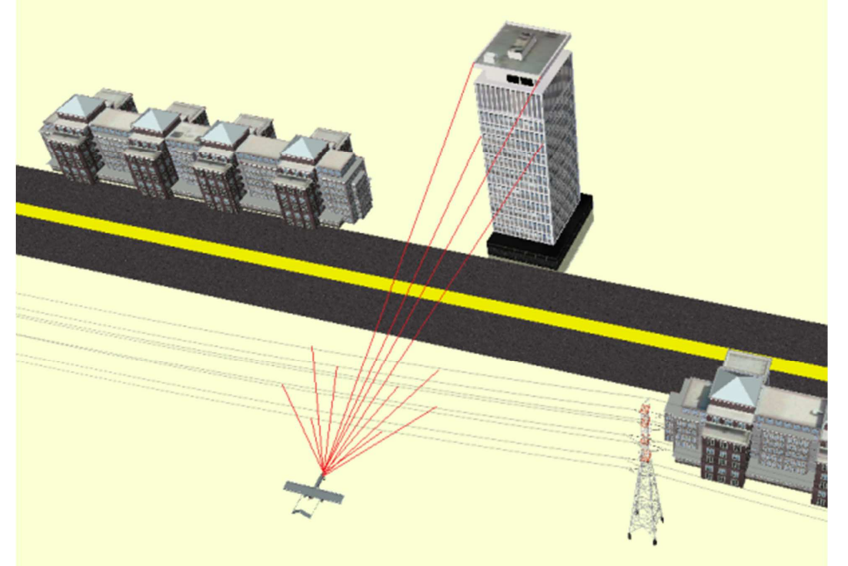

Fig. 12. Case study scenario. 
This is the author pre-publication version. This paper does not include the changes arising from the revision, formatting and publishing process. The final paper that should be used for referencing is:

S. Ramasamy, R. Sabatini, A. Gardi and J. Liu, "LIDAR Obstacle Warning and Avoidance System for Unmanned Aerial Vehicle Sense-andAvoid.” Aerospace Science and Technology (Elsevier), vol. 55, pages 344-358, 2016. DOI: 10.1016/j.ast.2016.05.020

Since wire targets are assumed to extend laterally, the trajectory characterised by the greater distance (and hence optimal in this case) in the one entailing a straight climb manoeuvre. The re-join trajectory is computed using pseudospectral optimisation techniques described in [29]. The UAV also avoids the extended target (building) by generating a smooth optimised trajectory from the safe manoeuvring point. These simulations were executed on a Windows 7 Professional workstation (64-bit OS) supported by an Intel Core i7-4510 CPU with clock speed 2.6 $\mathrm{GHz}$ and 8.0 GB RAM. The execution time for uncertainty volume determination and avoidance trajectory optimisation algorithms was in the order of $1.4 \mathrm{sec}$. Such an implementation makes it possible to perform real-time separation maintenance and collision avoidance tasks.

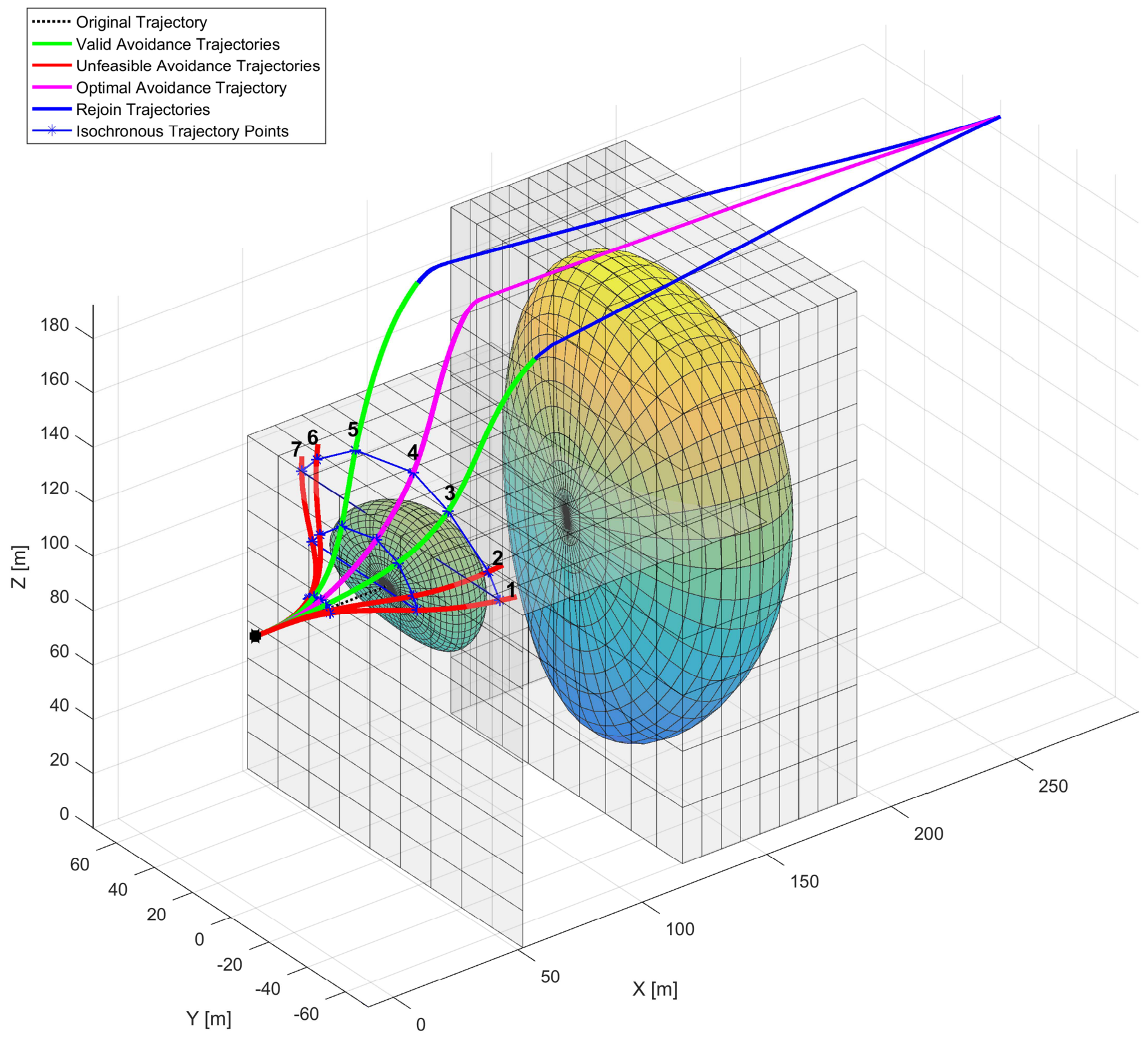

Fig. 13. Results of the avoidance trajectory generation algorithm.

\section{Further developments}

Fig. 14 shows synthetic display formats being developed for lowlevel flight applications (i.e., ground obstacles) [30]. In particular, Fig. 14-a depicts the Safety Line (SL), which connects the points of minimum pitch for safe obstacle avoidance at all azimuths. Fig. 14-b exemplifies the representation of Wires and Poles (WP) shaped obstacles, whereas Fig. 14-c shows obstacles of All Obstacles (AO) including the ones with bulk geometry (trees). Fig 14-d depicts the synthetic vision format integrating information from the LOWAS and from FLIR systems (Integrated LOWAS/FLS (ILF) format).

Future research activities will address synthetic formats for aerial obstacles display and the integration of LOWAS with FLIR and Night Vision Imaging Systems (NVIS), to exploit the available synergies in terms of obstacle/threat detection, recognition and identification [2, 31]. As part of the SAA system development activities, suitable hardware components and data fusion 
This is the author pre-publication version. This paper does not include the changes arising from the revision, formatting and publishing process. The final paper that should be used for referencing is:

S. Ramasamy, R. Sabatini, A. Gardi and J. Liu, "LIDAR Obstacle Warning and Avoidance System for Unmanned Aerial Vehicle Sense-andAvoid.” Aerospace Science and Technology (Elsevier), vol. 55, pages 344-358, 2016. DOI: 10.1016/j.ast.2016.05.020

techniques for cooperative and non-cooperative UAS SAA tasks will be developed, allowing a safe and unrestricted integration of UAS into all classes of airspace [29]. The SAA system can be integrated into the avionics compartment of existing UAV (Fig. 15) with a minimum impact on the payload capacity.

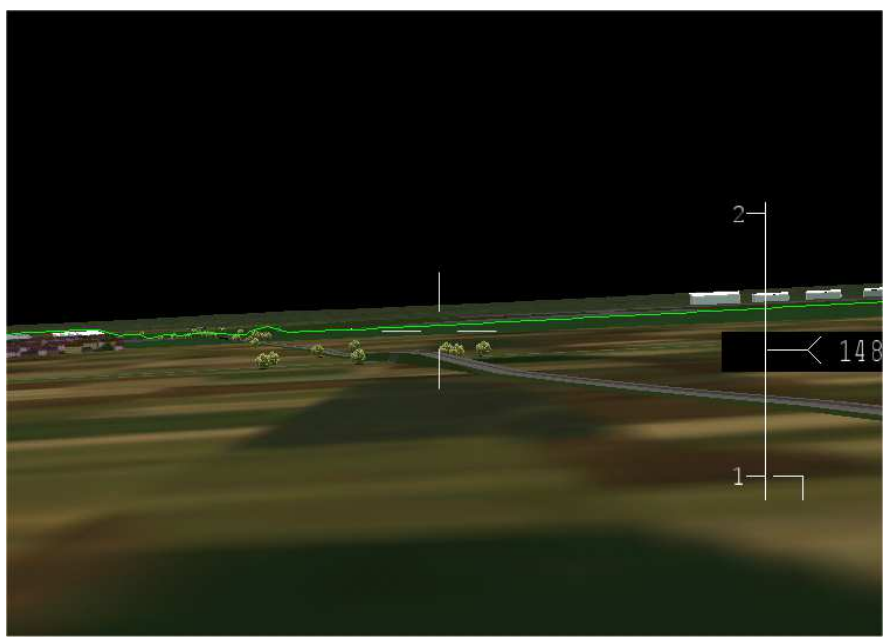

(a)

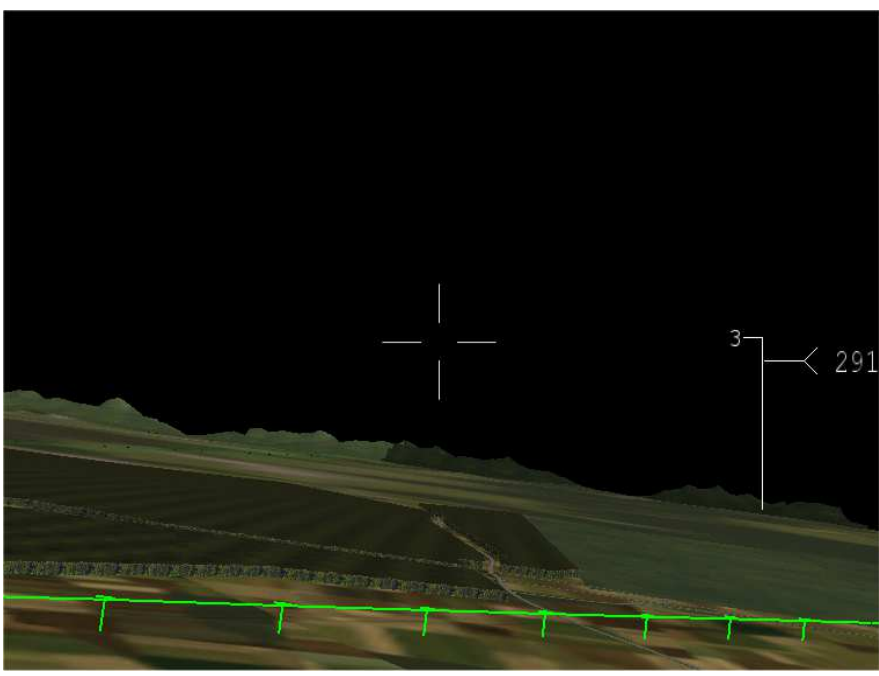

(b)

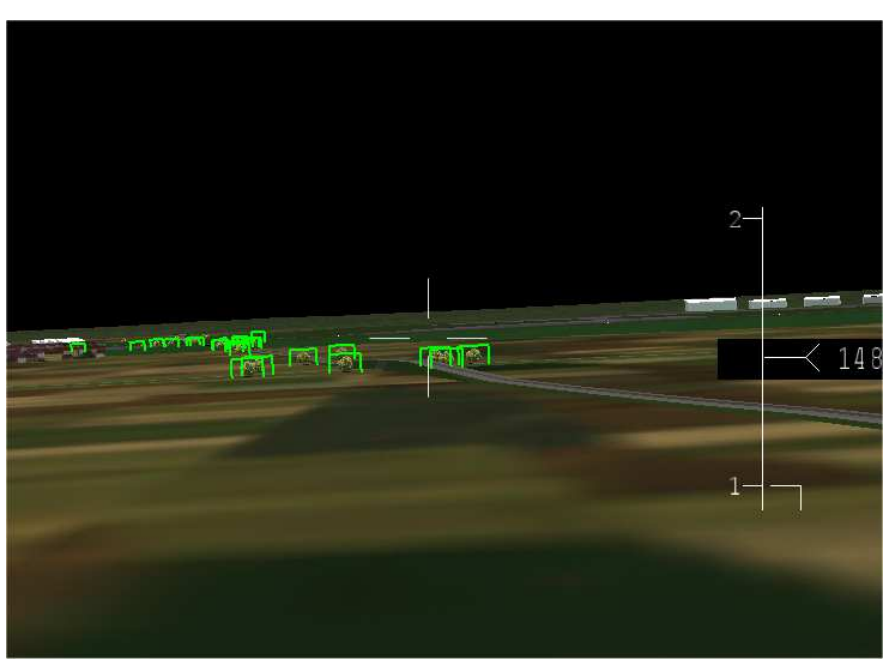

(c)

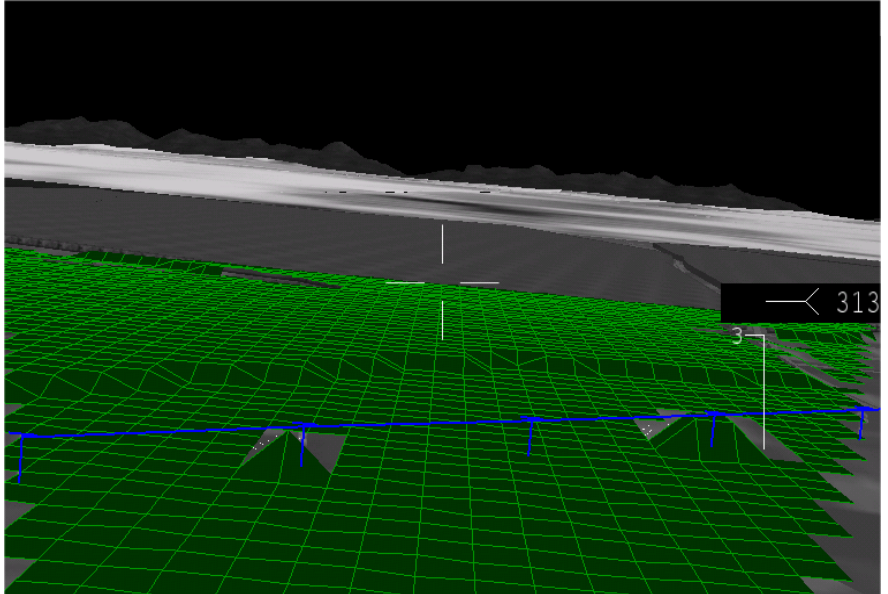

(d)

Fig. 14. Synthetic display formats: safety line (a), wires \& poles (b), all obstacles (c) and integrated LOWAS/FLIR (d).

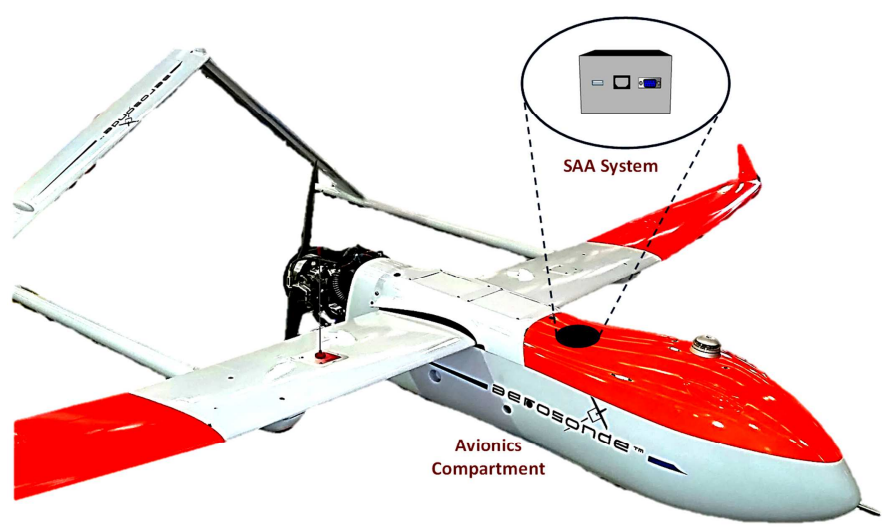

Fig. 15. SAA system integration in the avionics compartment of a smallsize UAV.

It is expected that this SAA system will allow:

- safely integrating UAS in all classes of airspace;

- operation of multiple manned and unmanned aircraft in close proximity to each other;

- the remote pilot and/or operator to supervise and supplement the execution of various SAA tasks;

- seamless integration with the ATM system.

Furthermore, the SAA system verification and validation schemes required to attain certification are being identified. Using suitable data link and signal processing technologies on the ground, a certified SAA capability will be a core element of future networkcentric ATM operations. The distinctive advantage that the presented SAA system offers towards certification is the capability of defining the safe-to-fly UAS envelope based on the available on board sensors or, alternatively, to identify the sensors required in order to achieve a certain predefined safe operational envelope for the UAS. 
This is the author pre-publication version. This paper does not include the changes arising from the revision, formatting and publishing process. The final paper that should be used for referencing is:

S. Ramasamy, R. Sabatini, A. Gardi and J. Liu, "LIDAR Obstacle Warning and Avoidance System for Unmanned Aerial Vehicle Sense-andAvoid.” Aerospace Science and Technology (Elsevier), vol. 55, pages 344-358, 2016. DOI: 10.1016/j.ast.2016.05.020

\section{Conclusions}

The research activities performed to develop a novel Laser Obstacle Warning and Avoidance System (LOWAS) for unmanned aircraft were presented. The LOWAS system is proposed as one of the core non-cooperative sensors in an integrated Sense-and-Avoid (SAA) architecture for small-tomedium size Unmanned Aerial Vehicle (UAV). The algorithms for computing the avoidance volumes associated with obstacles and for the generation of optimal avoidance trajectories were presented along with a representative simulation case study. Tailored display formats developed for the UAV remote pilot station were presented including Safety Line (SL), Wires \& Poles (WP), All Obstacles (AO) and Integrated LOWAS/FLS (ILF) formats. The demonstrated detection, warning and avoidance performances, determination of overall uncertainty volumes and avoidance trajectory generation algorithms ensure a safe avoidance of all potentially conflicting obstacles. The possible integration of LOWAS with other UAV tracking and navigation/guidance systems is currently being studied and future developments will focus on hardware/software development and flight test activities on small-size UAV.

\section{Conflict of interest statement}

The authors declare that this research was performed under no conflict of interest.

\section{References}

1. J. Gauci and D. Zammit-Mangion, Obstacle Detection around Aircraft on Ramps and Taxiways through the Use of Computer Vision, AIAA Guidance, Navigation and Control Conference (GNC 2009), Chicago, IL, USA, 2009.

2. R. Sabatini and M. A. Richardson, Airborne Laser Systems Testing and Analysis, RTO AGARDograph AG-300 vol. 26, Flight Test Instrumentation Series, Systems Concepts and Integration Panel (SCI126), NATO Science and Technology Organization, 2010.

3. X. Yu and Y. Zhang, Sense and Avoid Technologies with Applications to Unmanned Aircraft Systems: Review and Prospects, Progress in Aerospace Sciences, vol. 74, pp. 152-166, 2015.

4. S. I. Ali Shah and E. N. Johnson, 3D Obstacle Detection using a Single Camera, AIAA Guidance, Navigation and Control conference 2009 (GNC 2009), Chicago, IL, USA, 2009.

5. A. Moses, M.J. Rutherford and K.P. Valavanis, Scalable RADAR-Based Sense-and-Avoid System for Unmanned Aircraft, Handbook of Unmanned Aerial Vehicles, pp. 1895-1953, Springer, 2014.

6. K. R. Noth, Modeling and Simulation of a Ground based Sense and Avoid Architecture for Unmanned Aircraft System Operations, $11^{\text {th }}$ IEEE/AIAA Integrated Communications, Navigation and Surveillance conference: Renovating the Global Air Transportation System (ICNS 2011), Herndon, VA, USA, 2011, pp. O71-O79.

7. S. Ramasamy and R. Sabatini, A Unified Approach to Cooperative and Non-Cooperative Sense-and-Avoid, International Conference on Unmanned Aircraft Systems (ICUAS 2015), Denver, CO, USA, 2015.

8. K. Dalamagkidis, K.P. Valavanis and L.A. Piegl, On Unmanned Aircraft Systems Issues, Challenges and Operational Restrictions Preventing Integration into the National Airspace System, Progress in Aerospace Sciences, vol. 44, issue 7, pp. 503-519, 2008.

9. D.K. Douglas and S.P. Cook, Achieving Sense and Avoid for Unmanned Aircraft Systems: Assessing the Gaps for Science and Research, Handbook of Unmanned Aerial Vehicles, Springer Netherlands, pp. 1841$1855,2015$.

10. R. Sabatini and M. A. Richardson, A New Approach to Eye-safety Analysis for Airborne Laser Systems Flight Test and Training Operations, Optics and Laser Technology, vol. 35, pp. 191-198, 2003.

11. R. Sabatini, M. A. Richardson, H. Jia and D. Zammit-Mangion, Airborne Laser Systems for Atmospheric Sounding in the Near Infrared, SPIE 8433
Laser Sources and Applications, Photonics Europe 2012, Brussels, Belgium, 2012.

12. R. Sabatini, A. Gardi, S. Ramasamy and M. A. Richardson, A Laser Obstacle Warning and Avoidance System for Manned and Unmanned Aircraft, IEEE Metrology for Aerospace (MetroAeroSpace 2014), Benevento, Italy, 2014, pp. 616-621.

13. F. G. Gebhardt, High Power Laser Propagation, Applied Optics, vol. 15, pp. 1479-1493, 1976.

14. R. Sabatini and M. A. Richardson, Novel Atmospheric Extinction Measurement Techniques for Aerospace Laser System Applications, Infrared Physics and Technology, vol. 56, pp. 30-50, 2013.

15. T. Elder and J. Strong, The Infrared Transmission Of Atmospheric Windows, Journal of the Franklin Institute, vol. 255, pp. 189-208, 1953.

16. R. M. Langer, Report on Signal Corps Contract No. DA-36-039-SC72351, 1957.

17. W. E. K. Middleton, Vision through the Atmosphere, University of Toronto Press, 1952.

18. R. Sabatini, M.A. Richardson, A. Gardi and S. Ramasamy, Airborne Laser Sensors and Integrated Systems, Progress in Aerospace Sciences, 2015.

19. T.B. Sheridan, Adaptive Automation, Level of Automation, Allocation Authority, Supervisory Control and Adaptive Control: Distinctions And Modes of Adaptation, IEEE Transactions on Systems, Man and Cybernetics, Part A: Systems and Humans, vol. 41, no. 4, pp. 662-7, 2011.

20. Y. Zhou, Research on Physiological Monitoring Technology for Aviation Human Factor Certification, Shanghai Jiao Tong University, 2013.

21. H. Ayaz, B. Onaral, K. Izzetoglu, P.A. Shewokis, R. McKendrick and R. Parasuraman, Continuous Monitoring of Brain Dynamics with Functional near Infrared Spectroscopy as a Tool for Neuroergonomic Research: Empirical Examples and a Technological Development, Frontiers in human neuroscience, vol. 7, p. 871, 2013.

22. A. Gardi, R. Sabatini and S. Ramasamy, Multi-objective Optimisation of Aircraft Flight Trajectories in the ATM and Avionics Context, Progress in Aerospace Sciences, vol. 83, pp. 1-36, 2016.

23. S. Ramasamy, R. Sabatini and A. Gardi, Towards a Unified Approach to Cooperative and Non-Cooperative RPAS Detect-and-Avoid, $4^{\text {th }}$ Australasian Unmanned Systems Conference 2014 (ACUS 2014), Melbourne, Australia, 2014.

24. O. von Stryk and R. Bulirsch, Direct and Indirect Methods for Trajectory Optimization, Annals of Operations Research, vol. 37, pp. 357-373, 1992.

25. J. T. Betts, Survey of Numerical Methods for Trajectory Optimization, Journal of Guidance, Control and Dynamics, vol. 21, pp. 193-207, 1998.

26. J. Z. Ben-Asher, Optimal Control Theory with Aerospace Applications, Education Series, American Institute of Aeronautics and Astronautics (AIAA), Reston, VA, USA, 2010.

27. A. V. Rao, Survey of Numerical Methods for Optimal Control, Advances in the Astronautical Sciences, vol. 135, pp. 497-528, 2010.

28. M. Burston, R. Sabatini, A. Gardi and R. Clothier, Reverse Engineering of a Fixed Wing Unmanned Aircraft 6-DoF Model Based on Laser Scanner Measurements, IEEE Metrology for Aerospace (MetroAeroSpace 2014), Benevento, Italy, 2014.

29. A. Gardi, S. Ramasamy, R. Sabatini and T. Kistan, CNS+A Capabilities for the Integration of Unmanned Aircraft in Controlled Airspace, Proceedings of 2016 International Conference on Unmanned Aircraft Systems (ICUAS '16), Arlington, VA, USA, pp. 531-539, June 2016.

30. R. Sabatini, A. Gardi and M. A. Richardson, LIDAR Obstacle Warning and Avoidance System for Unmanned Aircraft, International Journal of Mechanical, Aerospace, Industrial and Mechatronics Engineering, vol. 8, pp. 62-73, 2014.

31. R. Sabatini, M. A. Richardson, M. Cantiello, M. Toscano and P. Fiorini, A Novel Approach to Night Vision Imaging Systems Development, Integration and Verification in Military Aircraft, Aerospace Science and Technology, vol. 31, issue 1, 2013. 